\title{
THz Rectennas and Their Design Rules
}

\author{
Mazen Shanawani ${ }^{*,+}+$ (D), Diego Masotti ${ }^{+}$(i) and Alessandra Costanzo ${ }^{\dagger}$ \\ D.E.I., University of Bologna, Viale Risorgimento 2, 40136 Bologna, Italy; diego.masotti@unibo.it (D.M.); \\ alessandra.costanzo@unibo.it (A.C.) \\ * Correspondence: mazen.shanawani@unibo.it; Tel.: +39-051-20-93139 \\ + These authors contributed equally to this work.
}

Received: 22 September 2017; Accepted: 1 November 2017; Published: 17 November 2017

\begin{abstract}
The increasing demand for more efficient energy harvesting solutions has urged research for better harvesting solutions than the presently-available ones. While p-n junction solar cells have become commercially widespread, they are expensive and suffer from poor efficiency figures hardly reaching $20 \%$. Other radiation-electricity converters such as rectennas have a theoretical limit in excess of $80 \%$. However, no efficient rectenna solution for the terahertz frequency band has been commercialized or presented in the academic literature. In fact, there are many obstructions to an efficient solution. The aim of this paper is to address the key points towards an efficient and commercially-available solution by briefly reviewing the relevant literature and so identifying five factors that should be addressed in order to reach an efficient solution.
\end{abstract}

Keywords: $\mathrm{THz}$ rectennas; tunneling diode; geometric diode; waste heat; transfer matrix method; QTBM model; non-equilibrium Green function model; Seebeck effect; rectenna detectors; nonlinear circuit

\section{Introduction}

In recent years, there has been an increasing demand to reduce the pollution of the environment to meet the stringent measures posed by the governmental bodies throughout the world. In 2020, the cutting back of greenhouse gases should be 20\% compared to 1990 levels [1]. In a related development, the British government's National Energy Efficiency Action Plan (NEEAP) set an even higher figure to meet for residential housing carbon emission, where a reduction by $31 \%$ compared to 1990 levels should be met [2].

Consequently, it becomes desperate to look for more efficient and, of course, less carbon-emissive energy sources that can stand up to these measures within this relatively limited time. It is, therefore, expected that the renewable energy sources such as wind and sun radiation can verily help with achieving this goal without producing carbon emissions. While there can be some good mechanical solutions to this issue, this article is concerned about the recent development in proposed electrical solutions to harvest energy in the $\mathrm{THz}$ region using antennas that receive AC energy and rectify the waveform using a nonlinear diode. This system is often referred to in the literature as rectenna.

The idea of harvesting radiation directly and converting into an electrical current has been around for many decades starting from the photovoltaic effect earlier referred to as the Becquerel effect and moving forward to optimize the photovoltaic cells and look for other alternatives to achieve better performance in addition to minimizing their production costs. The motivation to achieve this goal is what fuels the demand for increasing the research on rectennas. Corkish et al. [3] theoretically argue for rectennas, working in the solar frequency range, for which power efficiency in excess of $80 \%$ can be achieved. In fact, efficiency figures in excess of $90 \%$ have been reported by Brown [4] by using fast-switching diodes and optimizing the match between the antenna and the rectifying diode for his 
$2.45 \mathrm{GHz}$ rectenna system. However, as the operation frequency increases, many critical limitations come into play and limit the achievable efficiency to well below 1\% [5].

The aim of this paper is to review the past and present trials on rectennas focusing on the important parameters and figures of merit for researchers to consider in their endeavors to reach more efficient rectenna systems. In Section 2, a historical background to the current state-of-the-art situation is given starting from the discovery and development of the photoelectric effect. The pronounced Brown prototype of the rectenna helicopter [4] is, also, briefly discussed. Then, we move on to higher frequencies for energy harvesting from the naturally-available sources. Section 3 is dedicated to the relation between the operation frequency and the naturally-available sources' power levels. The correct choice for the theory of operation based on the frequency will be justified. Section 4 will elaborate on the different theories to explain and model the diodes. Their main features and drawbacks will be discussed with a follow up of the most recent amendments to them and to the available models for rectifier simulation. The models will be recognized based on the operation condition (i.e., with/without insulator illumination) and the suitable models for each diode design (i.e., tunneling and geometric diodes differ in their suitable models). Section 5 is dedicated to analyzing the impact of the incident signal power on the correct choice of the diodes. This is a very generic term and refers to the received signal amplitude, frequency bandwidth, the received signal polarization and the impact of these factors on the best modeling and diode structure. The thermal effect, which is always present at above zero temperatures is discussed in Section 6 with more focus on the available thermal effects such as the thermionic emission and Seebeck effect, which has a promising future for its use for rectenna detectors. Section 7 will address the future aspects for effective production of rectennas where mass production with minimized costs are the industry's primary concerns. In the end, a conclusion for this review will be drawn in Section 8.

\section{Historical Notes}

\subsection{Discovery of the Photovoltaic Effect}

The idea of harvesting sun or light radiation and converting it into electricity is not new, and it may be traced back in time to the nineteenth century in 1839 when Edmond Becquerel [6] noticed the effect of illuminating silver chloride inside an acidic solution, which resulted in the generation voltage and current. From that point on, many contributions took place to optimize the system, paving the way for a more efficient prototype by Bell Labs in 1954 [7].

The mechanism of the photovoltaic effect is relatively straightforward and has remained the same since the release of the prototype mentioned above [7]. As shown in Figure 1, a solar cell is made from a junction of $\mathrm{p}$ - and n-doped semiconductor material, whereas the p-type dopant pushes the Fermi level down closer to the valence band. On the other hand, the n-type dopant pushes the Fermi level closer to the conduction band above which electrons are freed from the hole bound. When a photon hits the semiconductor material and gets absorbed by an electron-hole pair (and has enough energy to free the electron from its hole pair), the electron is freed such that it moves into the conduction band (i.e., above the red line shown in Figure 1), where the electric field of the depletion region sweeps it away into the n-type side cathode. Similarly, the resulting free hole is also swept away by the same field, but into the opposite direction of the p-type side anode. The resulting effect is an electromotive force (EMF) that causes a potential difference between the anode and the cathode, which can also generate a current in a closed circuit and is represented in Figure 1 as $V_{O C}$ by the displacement of the Fermi level upwards in the n-type and downwards in the p-type. 


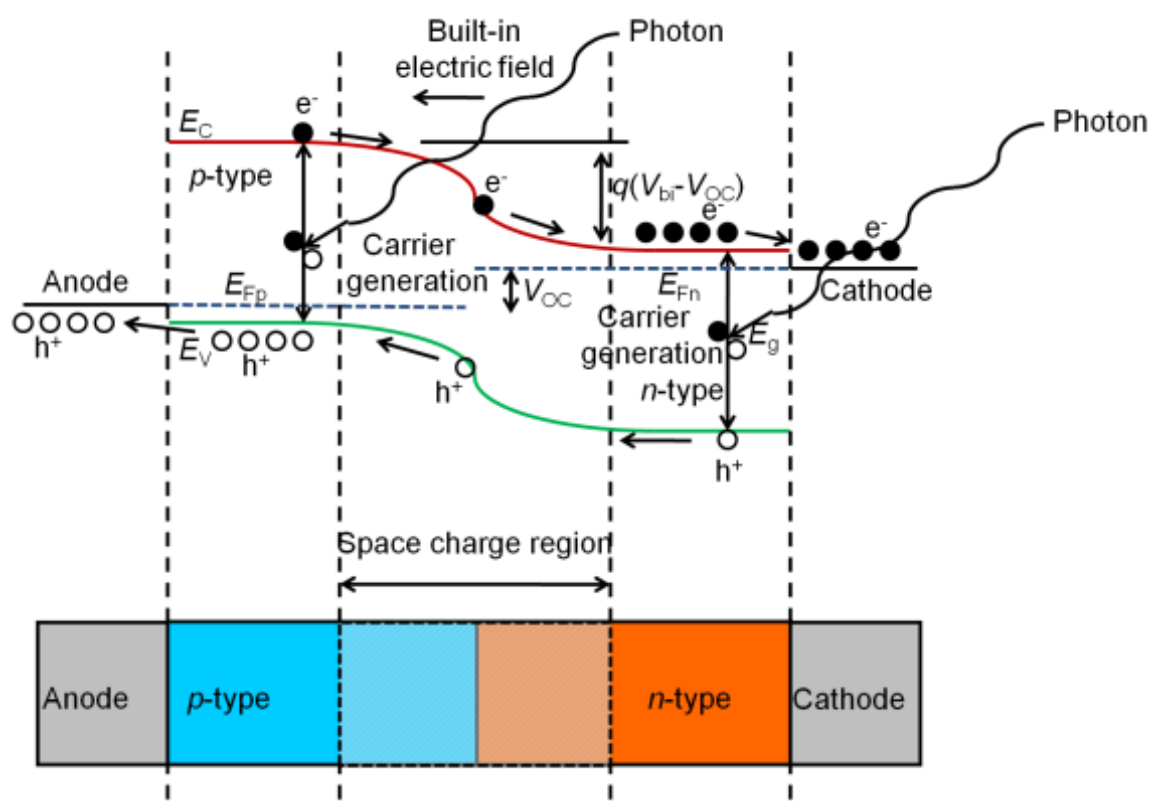

Figure 1. Energy-band diagram of a silicon p-n junction solar cell (Reproduced with permission from [8], Li Y., 2017).

Although the mechanism is simple, a critical power efficiency limitation is imposed (throughout the rest of this paper, the power efficiency is defined as the ratio of the DC power output yield to the input $\mathrm{AC}$ power delivered by the impinging radiation) because the required photon energy should be exactly equal to the energy required just to free the electron-hole pair. Clearly, if less energy is provided by the photon, then the bound will not be broken. Conversely, if the power provided by the photon is more than enough to free the electron, then the excessive energy will be wasted as heat into the semiconductor material. In other words, conversion efficiency can be optimal only for a narrowband radiation whose photon energy is just equal to that required to free the electron. Otherwise, the wideband nature of the solar radiation will yield sub-optimal efficiencies below the $44 \%$ Shockley-Queisser limit [9] calculated for a $1.1 \mathrm{eV}$ energy gap. In practical terms, this limitation is considered the major reason [10] as to why single junction solar cells have efficiency figures of around $20 \%$. This frequency-dependent behavior is not only limited to p-n junction solar cells, but also affects the rectennas' behavior in modeled prototypes as will become more evident in Section 5.

Notwithstanding all of the aforementioned facts, commercial systems are becoming more widespread, and some novel designs have managed to exceed $20 \%$ efficiency [11]. Thanks to multijunction solar cells [12], efficiencies up to $40 \%$ may be reachable. Although current commercial systems can reach up to $20 \%$ power efficiency, which is technically a poor figure, their production costs are still high with the current lowest production cost for a GaAs substrate reaching $\$ 150$ for a six-inch wafer [13]. It should be pointed out, however, that other alternatives, such as perovskite, may replace the typical solar cells with reported efficiencies in excess of $20 \%$ and much less expensive production costs [14]. Nevertheless, their lifetime is much shorter than that of their typical counterparts.

For all of the above, it might be more favorable to look for structurally different solutions that outperform the typical solar cells, preferably at a lower cost.

\subsection{Rectification of Electromagnetic Waves and Rectennas}

The concept of rectifying an electromagnetic wave into DC power may be attributed to William C. Brown who proposed a rectenna system in the first paper submitted to The Journal of Microwave Power in 1966 [15]. In his paper, Brown deploys "An array of Half-Wave Dipoles Terminated in a Bridge-Rectifier Array of Point-Contact Silicon Diodes." Although the diodes that Brown uses are 
assembled in a bridge form, the diagram of Figure 2 may be considered as a generic representation of rectennas based on its simple structure and the expandability of the model to higher frequencies.

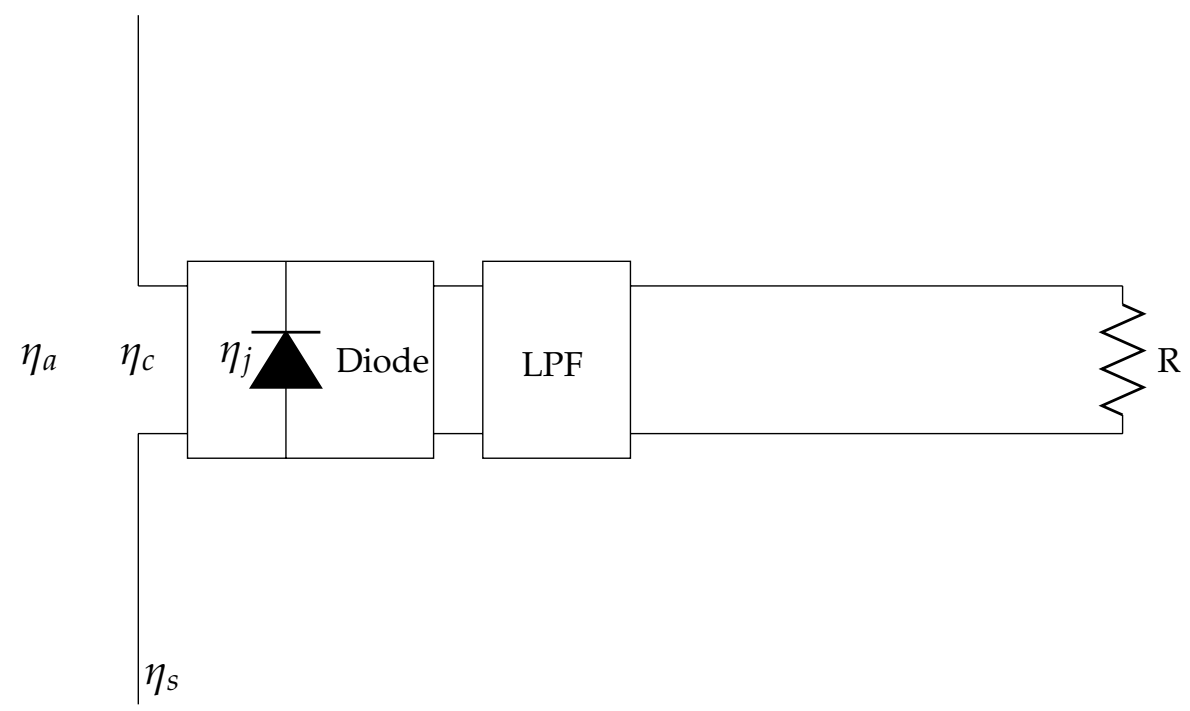

Figure 2. Generic circuit diagram for a rectenna system (Adapted with permission from [16], Springer, 2013).

Figure 2 shows the generic diagram of a rectenna system where the signal received by the antenna is directed to the diode, which rectifies the AC waveform into a DC signal that can deliver power to the load $\mathrm{R}_{\mathrm{L}}$. The low pass filter (LPF) unit will clearly restrict all components higher than the DC from passing into the load, thus keeping the AC part separated from the DC one. The letters $\eta_{a}, \eta_{c}, \ldots$ refer to the efficiencies of the respective parts and will be discussed later in Section 2.3.

In a later trial, Brown managed to increase the efficiency of his rectenna system to $92 \%$ [4]. This very good figure may be attributed to (a) the use of a Schottky barrier diode that features a fast switching time, as well as relatively low voltage drop and (b) the use of a filter between the dipoles and the diode network, which suggests that matching has been achieved between the two.

This work has drawn attention to the possibility of reaching significantly high efficiencies at high frequencies in the microwave region. For example, the work of Koert et al. [17] proved experimentally that an efficiency up to $72.5 \%$ is reachable at $35 \mathrm{GHz}$. Furthermore, more than $35 \%$ efficiency has been reported in the experiment of Chiou and Chen [18] in 2010 at the 94-GHz band for a dual-band design thanks to using filtering and matching techniques for a pair of Schottky diodes at the circuit termination points. The interested reader may refer to [19] (Figure 7) for a detailed representation of the recent work in this field.

The difference between rectennas and p-n junction solar cells lies in the fact that rectennas receive the radiation in its wave form as opposed to solar cells, which employ the quantum nature of light. However, quantum mechanics are frequently deployed in explaining the theory of operation of the rectifying diode as will become clear later on; thus paving the way for what is referred to as semiclassical models [20]. In these models, the radiation is perceived as an oscillating wave that interacts with the charge carriers whose behavior may be explained by quantum mechanics.

\subsection{Targeting Higher Frequencies}

The aforementioned efficiencies in Section 2.2 provide a good reason as to why rectennas might be favored over p-n junction solar cells. The experimentally-achievable efficiency is at least two-times that of the widespread solar cells. Moreover, their fabrication costs are generally less expensive. However, increasing the frequency into the $\mathrm{THz}$ range leads to many limitations. It is well known that the AC signal wavelength $\lambda$ is inversely proportional to the oscillation frequency $f$ such that 
fabricating the circuit components, be it antennas, filters, transmission lines or rectifiers, can be very challenging with dimensions in the $\mu \mathrm{m}$ range or even less. In addition to that is the onset of the lossy nature of metals and insulators, equally, at frequencies close to the visible range when their plasmonic features are not exploited [21]. To the authors' best of knowledge, there has been no attempt to implement a matching circuit for a rectenna system in the $\mathrm{THz}$ range.

It might be beneficial at this point to consider that $\mathrm{THz}$ rectennas are made up of two main parts as shown in Figure 2, that is the micro- or nano-scale antenna, often referred to in the literature as a nantenna [22] and the rectifier diode. This separation is helpful in terms of tracking the research progress of both parts.

Antennas used with rectennas have witnessed major progress thanks to computer modeling that facilitated the prototyping and analyzing process. Today, many free and licensed computer-aided design (CAD) packages are available and provide in-depth analysis of antenna models. Many prototypes have been built and tested in a real environment at $1-\mathrm{THz}$ frequencies and above [23,24]; Masotti et al. [25] have modeled a 84-THz antenna array.

Rectifiers, on the other hand, still have many challenges to efficiently function as energy harvesting devices in the $\mathrm{THz}$ range. Power efficiency figures are still very low to compete with those of regular solar cells. In 2003, Berland [5] noted in his technical report many reasons for not achieving high efficiency. He attributes the poor figures to weak antenna-wave coupling, mismatch between the diode and the antenna, the semi linear $I(V)$ curve of the rectifying diode and the difficulty to accurately reproduce the diode prototype, which would lead to unpredictable results.

Interestingly, many researchers are working to optimize the design of the rectenna system using different diode types without much detail about the difference between them. Moreover, there is more than one theory to explain the output of rectenna devices. For example, while tunneling is assumed to be the reason for the resulting current response of a rectenna diode in the Grover and Moddel model [26], Russer et al. [27] try to explain the behavior of the rectenna device based on thermocoupling between both antenna branches. Fumeaux et al. [28] attempted a rigorous approach trying to explain the studied diode behavior assuming a composite behavior of thermal and tunneling effects [28]. In order to address and prioritize the factors for not reaching an efficient $\mathrm{THz}$ rectenna system, Grover and Moddel [26] proposed the following efficiency Equation (1), such that the overall rectenna system efficiency is formed by four terms. While it is a conceptual equation, it helps having an in-depth understanding of the possible reasons of the poor efficiency figures of the recent rectenna trials:

$$
\eta=\eta_{a} \cdot \eta_{s} \cdot \eta_{c} \cdot \eta_{j}
$$

where $\eta_{a}$ represents the coupling efficiency of the antenna with the impinging electromagnetic wave. $\eta_{s}$ stands for the antenna efficiency. In other words, it represents the ratio of the delivered energy to the successive parts with respect to the energy already captured by the antenna. The ratio $\eta_{c}$ represents the coupling efficiency between the antenna and the diode. Hence, it reflects the importance of matching between the antenna and the successive parts the diode in this case. Finally, $\eta_{j}$ is the diode efficiency measured in $\mathrm{A} \mathrm{W}^{-1}$. Thus, it sets the unit of the final efficiency $\eta$ to $\mathrm{A} \mathrm{W}^{-1}$ because all other efficiencies are dimensionless.

This framework helps with viewing the recent work in this field such that developing the antenna or nantenna part is related to optimizing the values of $\eta_{a}$ and $\eta_{s}$. On the other hand, increasing the values of $\eta_{c}$ and $\eta_{j}$ happens only by using well-matched and efficient diodes; thus suggesting that the increased efficiency is the result of both actions. In order to bring more balance to the terms of (1), it might be favorable to increase the values of $\eta_{c}$ and $\eta_{j}$ to get a real improvement as compared to earlier findings. Indeed, the diode's poor efficiency has been addressed as the bottleneck towards reaching an efficient rectenna design in the $\mathrm{THz}$ region [29].

In fact, improving the diode efficiency and matching is related to many other factors, which will be discussed in more detail later on. In order to avoid confusion, these factors are categorized as shown 
in Figure 3. From the authors' point of view, the following reasons have a major impact on the rectifier diode efficiency:

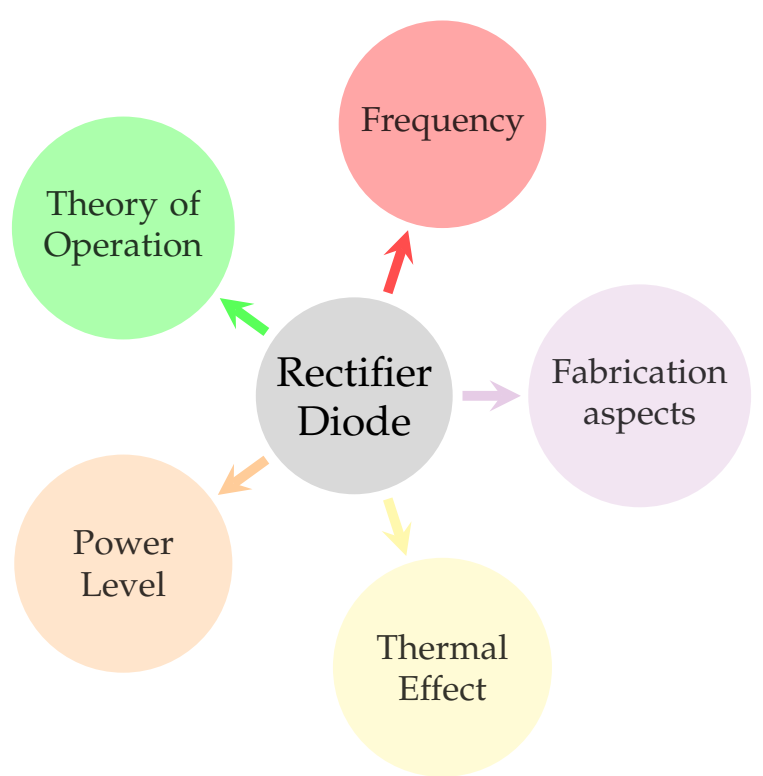

Figure 3. Factors affecting the rectifier diode efficiency.

- Frequency represents the frequency of the impinging electromagnetic wave on the antenna element. Recent trials in the $\mathrm{THz}$ range extend over a very wide frequency range for both modeled and realized systems with frequencies starting from $1 \mathrm{THz}$ [30], reaching the ultra violet range and exceeding $800 \mathrm{THz}$ [31]. Strictly speaking, over this very wide range, the proposed mechanisms and expected efficiencies for implementation vary largely as will be explained later.

- The theory of operation is a controversial issue for rectennas since there are many theories of operation and so many diode types that can be structurally different based on their different theoretical framework. The difference is not only between different diode types, but also extends to having more than one theory to explain the same diode behavior. One good example is the tunneling diode as mentioned earlier in this section. A review of the widespread theoretical frameworks will be provided in Section 4.

- Power level refers to the expected power level of the received signal, which can vary largely for irradiation intensities ranging from $60 \mathrm{~W} \mathrm{~m}^{-2}$ for ground radiation [32] up to more than $1000 \mathrm{~W} \mathrm{~m}^{-2}$ for visible light during daylight [33]. This factor can have a significant impact both on the design of the antennas and on the correct choice of the rectifying diode. Furthermore, it does not refer only to the signal input amplitude, but also largely depends on whether the signal is narrow or wide band, the coherence between different frequency components and even the distance between the source and the rectenna and how this can affect the coherence of the received wideband signal. Mashaal and Gordon [34] provide a detailed account of this issue as will be discussed later.

- Thermal effect: Although it might be argued that this factor is better considered with the theory of operation, the thermal effect is present in almost every diode application and can even take more than one form in the same application as will be seen later; the most common of which is the thermionic effect [35].

- Fabrication aspects: After a theoretical model is adopted, it would be realized using the available methods for fabricating the rectenna system at the nano-scale. This can be a challenging task, particularly, with small components whose dimensions are in the nm range. Although there are some promising prototypes efficiency-wise [36], which involves a vacuum clearance at the 
nanometer scale between the diode electrodes, realizing them in practice is a real challenge. Moreover, there are some prototypes whose $I(V)$ behavior becomes distorted with respect to simulation because of fabrication inaccuracies as reported by Conley and Alimardini [37].

\section{Frequency}

Based on their generic structure, rectennas are assumed to collect the radiation energy from the ambient environment. As for the microwave range up to a few hundreds of $\mathrm{GHz}$, there has been tangible progress in many fields. Theoretically, the problem here is usually approached from the antenna theory point of view such that the objective is to optimize the received microwave power and focus the transmitted power when possible. For frequencies above this range, there is usually little control over the radiating object such that the radiation may come out from any object around the rectenna device, be it walls, floor, hot surfaces or maybe visible light. Additionally, the reason for this radiation is just because the object has a temperature above $0 \mathrm{~K}$ where the matter particles are agitated, and so, they radiate energy at very high frequencies. It is, therefore, convenient to assume all surrounding objects to follow the black body radiation law. It is out of the scope of this paper to discuss the theoretical details of this physical phenomenon, and the reader may consult [38] for further information about its equation. Figure 4 shows the radiation intensity functionality regardless of the values with respect to wavelength $\lambda$. As the root locus of the intensity peak shows, when the radiating body temperature rises, the intensity peaks rise in amplitude and shift to the left, which represents an increase in the corresponding frequency $f$. For a radiating body whose temperature is $7000 \mathrm{~K}$, the intensity has a sharp peak at the highest visible wavelength violet color. Conversely, a $3000 \mathrm{~K}$ body has a much lower intensity peak and much wider spectrum, and the peak occurs within the invisible infrared range.

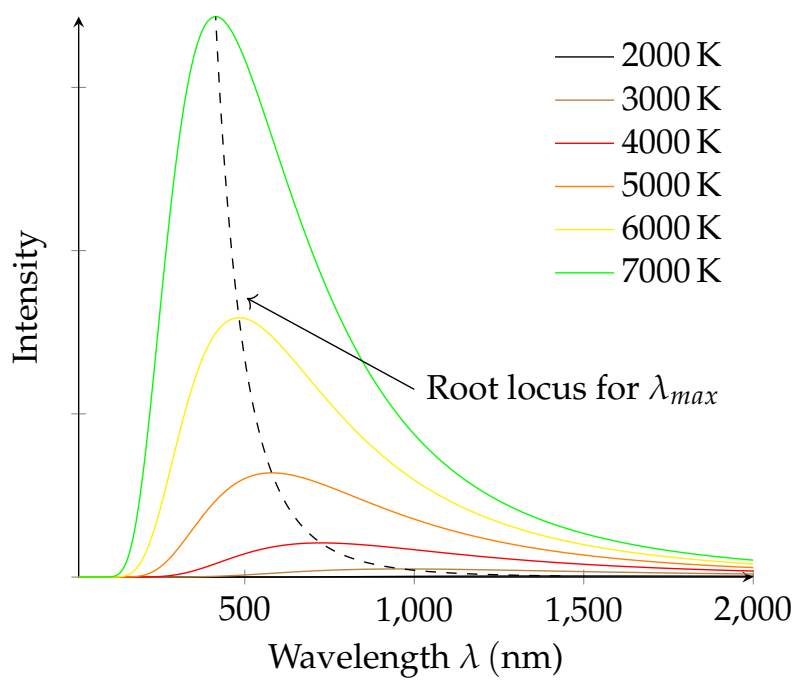

Figure 4. Black body radiation law curves for different temperatures. For the highest temperature $7000 \mathrm{~K}$, the intensity peak is high and sharp, while it tends to level out for lower temperatures as for the case of $2000 \mathrm{~K}$.

While harvesting energy from the sun can be very rewarding based on its high intensity, attempting frequencies as high as $800 \mathrm{THz}$ is quite challenging with the available technology. On the other hand, there are many other sources that provide a good deal of energy at lower temperatures, hence lower radiation frequencies, and have more realistic designs to attempt. For example, the radiated heat from hot surfaces such as those of hot engines, motors, chimneys, etc., is better harvested than being wasted into space, and they are quite abundant. This energy source is usually referred to in the literature as waste heat energy source [16]. 
Having said that, there have been some challenges such as their wideband nature, as seen in Figure 4, a very low power level that requires very low voltage drop for rectifying diodes, as well as their proximity to the harvesting rectenna in any future design, which in turn may lead to diminishing returns as will be shown in Section 5.2.1. In practical terms, if waste heat sources are assumed to have temperatures around $373 \mathrm{~K}$, their radiation intensity peak will be around $30 \mathrm{THz}$ according to Planck's radiation formula [38] and the corresponding wave length $\lambda=10 \mu \mathrm{m}$.

Although increasing the operation frequency opens up a new range for applications, the process is hampered by many challenges. Most importantly, the classical physics approach used for describing charge transition in semiconducting material fails to explain their behavior at extremely high frequencies when the radiation wave oscillates at much higher speeds than those of charge carriers. At these speeds, quantum approaches extend to explain the behavior based on Schrödinger's equation. Equally important is the effect of photo-assisted tunneling [39], which explains the behavior of electrons crossing a potential barrier illuminated by a radiation with frequency $f$. This effect starts to get noticed even before reaching the $1 \mathrm{THz}$ frequency as stated by Habbal et al. [40].

It is also important to point out that as the frequency increases, insulators' behavior can also change greatly. The dielectric constant values reported for many oxides used today in THz diodes' fabrication are contradictory. For example, niobium oxide, $\mathrm{Nb}_{2} \mathrm{O}_{5}$, has different reported values in [41-43]. Among many other factors, the frequency under which the material was tested plays a major role in its value.

\section{Theory of Operation}

As Figure 5 suggests, the categorization of different theoretical frameworks is not only according to the charge carrier transition mechanism, but also the suggested models under each category can have different application ranges. In all cases, they can be divided into three main categories; that is, tunneling, geometric and a hybrid mode, which shares some features of the previous two.

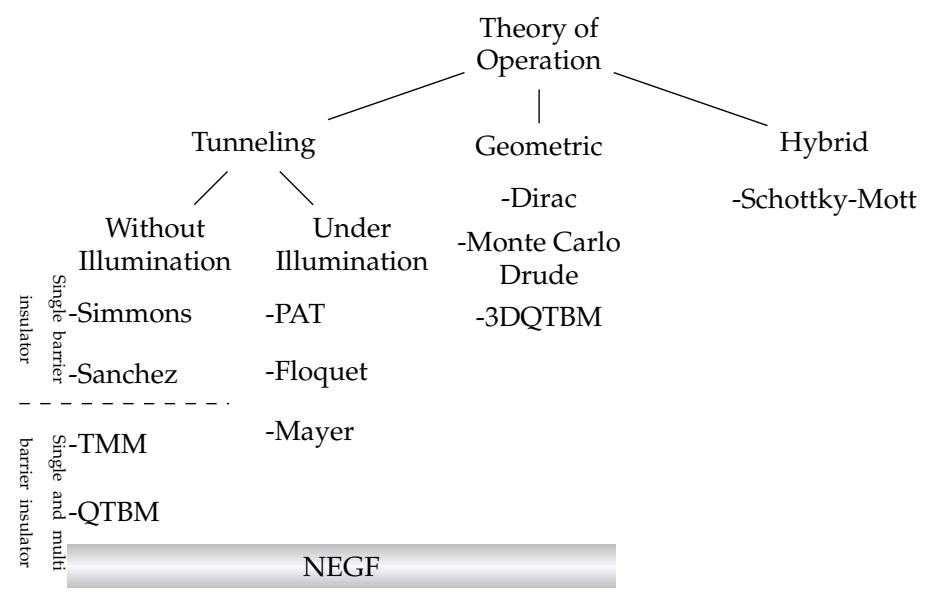

Figure 5. Categorization of the theory of operation.

\subsection{Tunneling}

The tunneling category represents the quantum mechanism that describes the transition of charge carriers based on the Schödinger wave equation, which can be written in its generic form as follows:

$$
\begin{gathered}
i \hbar \frac{\partial \Psi}{\partial t}=-\frac{\hbar^{2}}{2 m} \frac{\partial^{2} \Psi}{\partial x^{2}}+V \Psi, \\
\hat{H}=\hat{T}+\hat{V} \\
=\frac{\hat{\mathbf{p}} \cdot \hat{\mathbf{p}}}{2 m}+V(\mathbf{r}, t)=-\frac{\hbar^{2}}{2 m} \nabla^{2}+V(\mathbf{r}, t),
\end{gathered}
$$


In (2), $\hbar$ stands for Planck's constant divided by $2 \pi, m$ is the particle effective mass, $i$ is the imaginary number, $V$ is the potential energy the particle has and $\Psi$ is the wave function. The $\hat{H}$ shown in (3) represents the Hamiltonian operator, which consists of both the static energy $V(\mathbf{r}, t)$ and the kinetic energy operator represented by $\hat{T}$. Although we will not attempt any theoretical work regarding these equations in this paper, it is important to understand some of their implications because they impact the adopted models for simulation as will become clear later. The simulation approaches may be divided, based on (2), into simulations for a time-independent wave function and a time-dependent wave function; which correspond to the no illumination and the under illumination cases shown in Figure 5, respectively.

For this category and regardless of the tunneling theory, the procedure is the same such that the Schrödinger equation is used to calculate the transmission probability of the electron, and then, the current density can be found from multiplying this probability by the number of available electrons. This theoretical framework is well explained in [44,45]. The reader, while reading other literature, might encounter tunneling types such as Fowler-Nordheim tunneling (FT), resonant tunneling (RT), step tunneling (ST), direct tunneling (DT), etc. These are types that obey the same tunneling theoretical framework and rely on insulators thicknesses, biasing voltage and potential barrier height. So, we prefer not to explain them discretely as they can happen in any tunneling diode based on the aforementioned parameters. The current is then simply the current density multiplied by the contact surface area. Although many models adopt this approach, Heiblum et al. [46] argue that the tunneling effect happens at the surface edges rather than the center part of the junction. The experiment result of Fumeaux et al. [28] as shown in (Figure 29b) of their work supports this assumption where the polarization-dependent response component $V_{p}$ seems to level out with increasing the contact area in an asymptotic approach.

Following Figure 6, the diagram shows the energy band of one type of tunneling diode, the metal-insulator-metal (MIM) diode; when the diode is forward biased, the electron will tunnel from the higher barrier region identified as $\Phi_{L}$, to the lower barrier region identified as $\Phi_{R}$. This is an asymmetric diode where the barrier heights are different at both ends, and this happens when two metal types are used. Conversely, a symmetric diode utilizes electrodes of the same metal at both ends. It is important to notice that the tunneling will occur anyway, with or without an illuminated junction, as long as there is enough forward bias represented as displacement between Fermi levels of the left and right regions by $e V_{D}$ and in the same direction shown in the figure. Illumination of the junction would affect the tunneling phenomenon differently as experimentally represented [40].

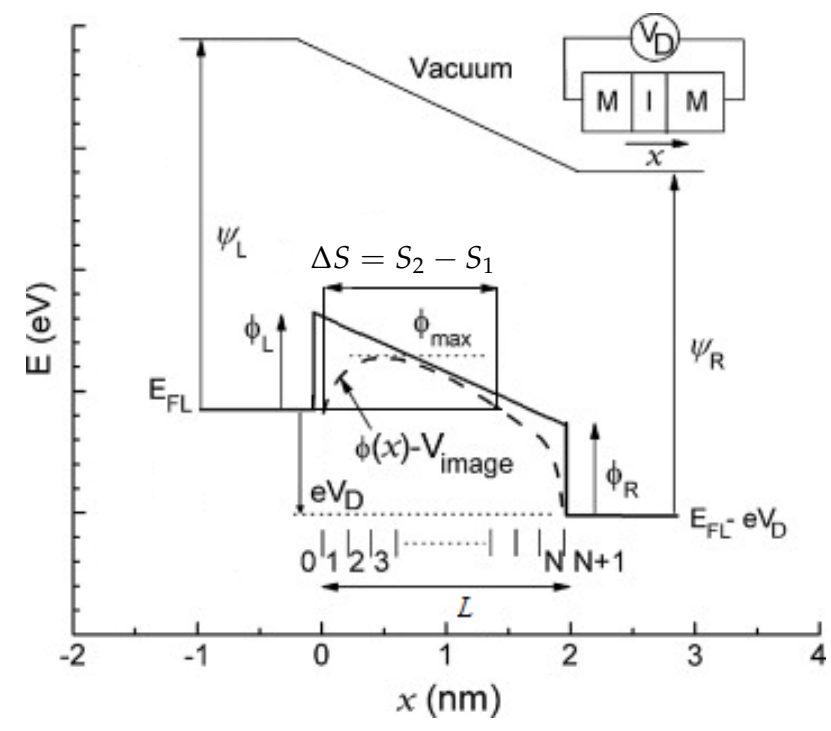

Figure 6. Energy band diagram of a metal-insulator-metal (MIM) tunneling diode (Adapted with permission from [47], Springer, 2012). 


\subsubsection{Without Illumination}

When a charge carrier electron is expected to traverse through a tunnel, the likelihood of this occurrence is a function of the wave equation denoted in (2). However, the oscillating potential energy component of the electron imposed by the outside voltage will be independent of time if no outside photon (illumination, in other words) is encountered across the barrier. This is usually referred to as no illumination or the dark current situation. It would also yield a simplified solution to (2) since $V(\mathbf{r}, t)$ becomes $V(\mathbf{r})$. Having said that, finding an accurate solution to the Schrödinger equation has always been a challenge, and there are many proposals with different features that have been tried so far to approach an accurate solution to it. Following is a list of the commonly-used approaches in the literature:

Simmons model:

This model has been proposed by John Simmons [44] to find the $I(V)$ curve of a diode consisting of a thin insulating film between two metal electrodes. In his paper, Simmons assumes a potential barrier that takes into account the image forces [48] and formulates its approximation using a continuous function. The resulting current of the applied voltage is calculated by finding the probability of an electron passing through the tunnel, using the Wentzel, Kramers and Brillouin (WKB) approach [49], and multiplying it by the number of the available free electrons. The likelihood function is developed form the Schrödinger equation and is shown in (4) assuming that the electron transfer happens only in the $x$ direction; where $h$ is Planck's constant, $m$ is the electron's effective mass, $V(x)$ is the potential difference between the two electrodes and $E_{x}$ is the energy component of the incident electron in the $x$ direction. The presence of the image forces lowers the potential barrier as shown by the dashed curve in Figure 6. The intersection between this curve and the higher Fermi level of the cathode identifies the potential tunnel width $\Delta S=S_{2}-S_{1}$ which is smaller than the physical tunnel width of the trapezoid $-L$ in Figure 6-which is the insulator thickness; thus facilitating the tunneling of electrons. The presence of the image forces is largely affected by the dielectric value of the insulating material such that lower values lead to lower barrier heights. Thus, when a higher response is needed, it is favorable to use insulators with a low dielectric value.

$$
D\left(E_{x}\right)=\exp \left\{-\frac{4 \pi}{h} \int_{s_{1}}^{s_{2}} \sqrt{2 m\left(V(x)-E_{x}\right)} d x\right\},
$$

The major drawback of this model is that it leads to inaccurate current values many orders of magnitude lower than the experimental results. This has been noted by Chin et al. [50] where the metal work function $\Phi_{M}$ values used in the model to give comparable results to measurements are lower than those found by direct physical measurements. However, curve fitting may by used with the model equation and leads to good agreement with measurements. Moreover, the value difference between the fitted work functions is equal to that of measured values. The reader may consult [50] (Table I-II) for further information.

There have been many trials to improve the performance of the Simmons model. In his Master's thesis, Koppinen [51] tries a rigorous mathematical framework to improve the performance of this model, but the resulting model also needs curve fitting to experimental results; in which case, there is little point to optimizing this model. The reason for this behavior, as suggested by Koppinen, is the unphysical divergence of the barrier at the metal surface. Albeit that the model he presents overcomes the discontinuity problem of the potential barrier curve between the metal-insulator interfaces, it still needs curve fitting. Grover and Moddel [47] state that the WKB approach used by the Simmons model gives different results with respect to other approaches at lower barrier heights. Another drawback that this model suffers from is that the model is primarily a single insulator layer model as opposed to multi-insulator structures where more than one insulating layer is laminated in the diode structure. It requires a complicated mathematical work to account for multiple insulators with little output expected from this model. 
Although the model has many drawbacks and is kind of dated, it is still quite frequently cited in the literature. It is usually used as a reference model to compare to other more complicated models. Sanchez model:

This model was proposed in the late 1970s [45] with a more systematic approach to rectennas. In this model, Sanchez et al. focus on the best practice to optimize the power transfer between the antenna and the diode. It has been presented for infrared detection, as noted in their abstract, such that the diode is assumed nonlinear, the current of which has a third order polynomial functionality with respect to the voltage drop across the diode. Calculations are then made to optimize the power transfer between the antenna and the diode. The aim of the math is to find the relationship between incident power level and the physical and geometric characteristics to achieve maximum power transfer; again, using the same WKB model shown in (4) and used by Simmons. As the model is presented for detection, special attention is given to its noise performance with the introduction of the noise equivalent power (NEP) figure of merit.

To compare between their model expectations and the results of an experiment they performed using a $\lambda=377 \mu \mathrm{m}$ laser, they measured the dependence of the antenna efficiency represented by $\eta_{0}$ on the focused laser beam incidence angle $\theta_{f}$ and found that $\eta_{0}=f\left(\theta_{f}^{2}\right)$, which is consistent with the effective aperture concept of the antenna theory.

Lastly, the model is quite frequently quoted in the literature and represents a more realistic approach to a systematic rectenna despite the fact that it is structurally limited to single-layer insulators. Moreover, the model indirectly suggests not to apply it to an insulator thickness more than $10 \AA$ as mentioned in [45] (Section III), and so, it has fewer application fields. Furthermore, the best achievable coupling efficiency was found to be $1 \%$ as shown in [45] (Figure 8 ) using a $377 \mu \mathrm{m}$ laser. It is worth noting that a "weak bolometric response" was observed.

Transfer matrix method:

This is also referred to in the literature as TMM. Technically, the transfer matrix method has been applied in other fields such as in the optics, acoustics and electromagnetic fields [52]. Jonsson and Eng [53] applied it to Schrödinger's equation deploying Bastard's approximation [54]. The concept is to use numerical modeling on a physical problem such that the problem structure is divided into smaller parts within which the wave equation, the potential energy, as well as the particle effective mass are assumed constant. Then, any change in any of these values is accounted for gradually when moving from one cell to another. Figure 7a shows the potential barrier value such that the whole curve is divided into many parts, and each part has a constant potential value throughout the containing cell.

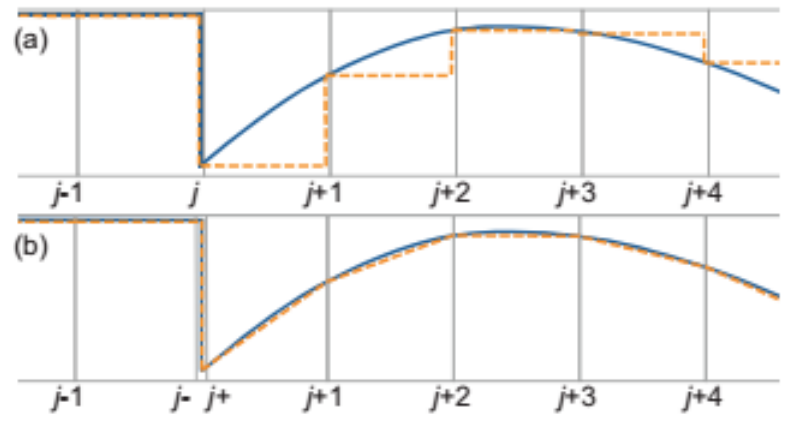

Figure 7. Energy-band diagram of a silicon p-n junction solar cell (Reproduced with perimission from [55], IEEE, 2009).

To simplify the problem, it is assumed that:

1. The modeled structure is a one-dimensional structure such that the modeled diode metal insulator interface infinitely extends and that the potential value changes only in one direction normal to the interface. 
2. The Schrödinger wave equation time is approximated using Bastard's assumption [54] in which case all the rapid changes of the wave function with respect to time are neglected, and the wave function becomes as shown in (5). This simplification allows one to treat the wave function as a time-independent parameter.

$$
u(\mathbf{r}, t)=\Psi(\mathbf{r}) \cdot e^{-2 \pi i E t / h},
$$

3. The simplified wave function, which is now only space-dependent, is further divided into two components, according to Euler's formulas. As shown in (6) for the $j$-th cell. $A_{j}$ and $B_{j}$ can be determined through the boundary conditions of the wave function, and $p_{j}(x)$ is a complex function whose value can be determined from [53] (6).

$$
\Psi_{j}(x)=A_{j} \cdot e^{p_{j}(x)}+B_{j} \cdot e^{-p_{j}(x)},
$$

Yet further, the $A_{j}$ and $B_{j}$ values of adjacent cells can be related using the matrix relation depicted in (7). Applying this framework for all successive cells will lead to the solution of the considered physical problem. Thus, the tunneling likelihood for an electron $D\left(E_{x}\right)$ is found, then the process will be similar to that adopted by Simmons [44].

$$
\left[\begin{array}{l}
A_{j+1} \\
B_{j+1}
\end{array}\right]=M_{j} \cdot\left[\begin{array}{l}
A_{j} \\
B_{j}
\end{array}\right]
$$

After solving this set of equations, the transmission probability of an electron $T\left(E_{x}\right)$ can be found from (8), where $k_{0}=\sqrt{2 m_{e} m_{L} e E_{x}} / \hbar$ and $k_{N+1}=\sqrt{2 m_{e} m_{R} e E_{x}} / \hbar$ such that $m_{e}, m_{L}$ and $m_{R}$ are the electron effective mass in the insulator, left electrode metal and right electrode metal, respectively.

$$
T\left(E_{x}\right)=\frac{k_{N+1}}{k_{0}} \cdot \frac{\left|A_{N+1}^{+}\right|^{2}}{\left|A_{0}^{+}\right|^{2}}
$$

The transfer matrix method has gained increasing interest over the last two decades starting from its introduction by Jonsson and Eng [53]. The algorithm is inherently simple as it uses the widespread TMM concept and applies it to a simplified version of the Schrödinger equation. Even so, its performance is superior to that of WKB in particular for low barrier MIM diodes [47]. Another added value for this method is its adaptability to simulate multi-insulator diodes as shown by Hashem et al. [56]. Moreover, the algorithm simulation output is in close agreement with the more computer-intensive algorithms such as the quantum transmitting boundary method (QTBM) [47] and the non-equilibrium Green function method (NEGF) [56], which will be encountered later on.

On the other hand, the method has some drawbacks that have been referred to much earlier. Jonsson and Eng [53] state that the accuracy of this method is three to four computer digits using the computer technology of the time. Trials have been performed to improve its accuracy by introducing a linear potential barrier increase inside the cell instead of a constant value [55], as shown in Figure 7b. This can also be referred to as the Airy function transfer matrix method (AF-TMM) [56], which gives identical results to that obtained by the NEGF algorithm as stated by Hashem et al. [56] for their model.

Another criticism of this method is the dependence on Bastard's approximation such that the wave function envelope approach has been postulated rather than getting derived from the microscopic Schrödinger equation [57].

Quantum tunneling boundary method:

Also referred to as QTBM, it was presented by [58]. It also relies on a time-independent Schrödinger form. Another simplification is made by assuming that the voltage value does not change along the leads that connect the device to the outside world. There are other assumptions that are imposed to simplify the Schrödinger solution. However, it solves for the equation in a 
two-dimensional approach, whereas the device is divided into smaller mesh cells, and the wave equation value inside the device is the main target to solve for in the algorithm as shown in (9).

$$
\Psi_{0}(\mathbf{r})=\sum_{i} \Psi_{i}(\mathbf{r}) \phi_{i}(\mathbf{r}),
$$

where $\Psi_{0}(\mathbf{r})$ is the wave equation value inside the device, the diode insulator in our case, $\Psi_{i}(\mathbf{r})$ is the wave function value inside cell iand $\phi_{i}(\mathbf{r})$ is the $\mathbf{r}$-dependent global shape function that takes the value of one inside the cell $i$ and zero in all other cells to suppress the values of $\Psi_{i}(\mathbf{r})$ outside their assigned cells. The problem is defined as solving for the vector of unknowns $\Psi_{i}(\mathbf{r})$, which is done through a system of linear equations as shown in [58] (54). The algorithm has many interesting features. Based on the concept it was built on, it is a multiport simulation algorithm, and so, it is ready for more complicated device shapes with more than one port input. The simulation output can provide more location-based information because the wave function is described by two dimensions. In the simulation performed in [58] (Figure 4a), the figure shows the wave function value surface plot of a rectangular cavity along a quantum waveguide. The authors in [59] provide another development to the algorithm to account for three-dimensional modeling for band-to-band tunneling in a nanowire. Three-dimensional QTBM simulation, also referred to as 3DQTBM, opens up better flexibility and accuracy options for the method.

In spite of the benefits of this algorithm, it is more computer intensive than the predecessor and still time independent as opposed to NEGF as will be shown below.

Non-equilibrium Green function:

This is frequently quoted in the literature as NEGF. The algorithm attempts to solve the wave function, without involving directly the Schrödinger equation, using the following definition for the Green function as shown in (10) taken from [60].

$$
(E-\hat{H}) G(E)=\mathbb{I},
$$

Assuming a wave function, the time independent Schrödinger equation can be expressed as:

$$
\hat{H} \Psi(\mathbf{r})=E \Psi(\mathbf{r})+V,
$$

where $V$ is the small perturbation introduced to help find a solution to this equation according to perturbation theory [61]. Now, the wave function can be found directly from (12):

$$
\Psi(\mathbf{r})=-G(E) V,
$$

The theoretical framework is rigorous and will not be attempted here. The algorithm is frequently quoted for its accuracy [56,62]. An outstanding feature of this modeling scheme is that it can be applicable to "uniform illumination" as stated by Datta and Anantram [63] where the oscillating field is expressed as $V(z, t)=V(t)=V_{0} \cos (\omega t)$ or if it takes a phase-dependent form, such that $V(z, t) \sim V_{0} \cos (\omega t-q z)$. More importantly, it is applicable to other devices than tunneling ones such as the geometric diode simulated by Zhu et al. [64]. Yet further, the algorithm can account for time-modulated potentials, and so, it becomes extendible for time-dependent equations [63] as shown in Figure 5.

On the contrary, its complicated math makes it computer intensive since it can be used to describe tunneling, non-tunneling and time-dependent voltage scenarios.

\subsubsection{Under Illumination}

The output of many experiments [40], Tucker, J.R., et al. [39] shows that the tunneling diodes behave differently under illumination as compared to their behavior without illumination. This difference in behavior can be expressed in quantum terms such that illumination can be considered 
as an oscillating potential or perturbation in the Hamiltonian function. Many approaches to solve this complicated Schrödinger problem have been proposed where each of them tries to approach the problem in a different manner, and the most frequent ones may be briefly reviewed here.

Tien-Gordon:

This effect was first introduced in [65] and is also referred to as photon-assisted tunneling (PAT) quite frequently in the literature $[39,66]$. In a similar style to [54], it assumes that the time-dependent wave function can be described as shown in (13).

$$
\Psi(\mathbf{r}, t)=f(\mathbf{r}) \cdot e^{\frac{-i E t}{\hbar}},
$$

where $\Psi(\mathbf{r}, t)$ is the modeled wave function, $f(\mathbf{r})$ is a spatial function, $E$ is the quasi-particle energy, $i$ is the imaginary number and $\hbar$ is the reduced Planck's constant. The introduction of an oscillating perturbation into the Hamiltonian results in the following Equation (14).

$$
\hat{H}=\hat{H}_{0}+e V \cos \omega t,
$$

Tien and Gordon [65] support their space-independent perturbation approach by the assumption that the wave function drops very sharply at both ends of the tunnel, so the interaction with the electric field is quite small. Based on that, they put the following form for the wave equation as shown in (15).

$$
\Psi(\mathbf{r}, t)=f(\mathbf{r}) \cdot e^{\frac{-i E t}{\hbar}}\left(\sum_{n=-\infty}^{n=+\infty} B_{n} \cdot e^{i n \omega t}\right)
$$

Working out some math, they find the coefficients $B_{n}$ to be $B_{n}=J_{n}\left(\frac{e V}{\hbar \omega}\right)$ where $J_{n}$ represents the $n$-th order Bessel function of the first kind. Without delving too much into this math, the current value for a tunneling device under illumination is found to be dependent on both the photon energy represented by its frequency $\omega$ and the oscillation amplitude $V$. The following current equation is found from the Tien and Gordon [65] model and is frequently used to explain the experiments' outputs up to a few $\mathrm{THz}[39,66]$ :

$$
I_{\text {illum }}=\sum_{n=-\infty}^{n=+\infty} J_{n}^{2}\left(\frac{q V_{w}}{\hbar \omega}\right) \times I_{\text {dark }}\left(V_{D}+n \frac{\hbar \omega}{q}\right)
$$

where $I_{\text {illum }}$ is the resultant illumination current, $q$ is the particle charge, the electron charge in this case, and $V_{D}$ is the DC voltage across the tunnel. The terms are summed from $-\infty$ to $+\infty$ to scan all the possible wave function modes. In physical terms, the illumination current is the composite result of emitting and/or absorbing photons with frequency $\omega$ such that the $n<0$ terms represent the absorption of photons and $n>0$ represents their emission. The amplitude of the Bessel function for each term represents the likelihood of the corresponding occurrence (i.e., a photon gets absorbed/emitted). Another issue is how many terms shall be included in a calculation? This by far depends on the value of the $n$-th Bessel function with respect to the fundamental one where the voltage amplitude has a major impact. Empirically, Joshi and Moddel [66] use values $n=-1,0,1$ in their model. Therefore, it makes sense to deploy more Bessel terms for higher oscillation amplitudes.

Contrary to the easier approaches of tunneling under no illumination, this concept results in a nonlocal functionality of current to voltage where the notation $I(V)$ becomes no longer accurate because it represents an instantaneous dependency; whereas the current $I$ depends on the current and previous values of voltages in this model. The functionality represented in (16) is in the frequency domain such that the current harmonics are found instead of its time-dependent value, which is attempted by Tucker and Feldman [39] and takes a more complicated form. 
Another notable issue in this model is that a single frequency oscillation generates multi-mode wave functions. This is connected to the findings of other more sophisticated models of the wave equation.

It should be noted, however, that Tucker and Feldman [39] state that more than single tone currents can be found out from this model, i.e., current components for $\omega, 2 \omega, 3 \omega, \ldots$. However, the only distinguished current component is the fundamental with frequency $\omega$ for practical reasons, as they explain. Equally important, the most prominent reason is that the cut off frequency is much lower than the second harmonic such that the diode capacitance appears as a short circuit to these higher-order current components. In other words, although the diode is considered a nonlinear device, only the first harmonic should be accounted for in any nonlinear simulation as the higher order harmonics have more complicated notation and much less impact.

In the end, this model has been in use and quoted many times due to its simplicity and the good correspondence it gives with experiments up to a few $\mathrm{THz}$ frequency. Having said that, the assumption of (13) has been disputed especially for higher frequencies and more complicated structures.

Other models:

To reach a more precise model of the time-dependent wave equation, there have been many introduced models in the literature. The following just reviews the most frequently-cited ones. For example, the Floquet model [67] exploits the case when the oscillation is a single frequency such that the Hamiltonian is periodically perturbed $\hat{H}(t)=\hat{H}(t+T)$ where $T$ is the perturbation period, then it can be proven that the wave equation solution of the Schrödinger equation is as follows (17).

$$
\Psi(\mathbf{r}, t)=e^{-i \cdot \epsilon_{r} t} \cdot \Phi_{\alpha}(\mathbf{r}, t),
$$

Whereas the $\epsilon_{r}$ is the Floquet characteristic exponent or quasi-energy [68], $\Phi_{\alpha}(\mathbf{r}, t)$ are the Floquet mode obeying functions such that $\Phi_{\alpha}(\mathbf{r}, t)=\Phi_{\alpha}(\mathbf{r}, t+T)$.

Another noticeable attempt is that of Truscott [69] who introduces a space-time coordinate transformation to solve for the wave equation and decompose its terms as shown in [69] (2). This transformation is claimed to improve accuracy over the models that adopt a spatially-uniform time-dependent field approach. Similar to Datta and Anantram [63], the algorithm can account for phase-change oscillating barriers with linear and parabolic relationships. The interesting output of this model is that "the onset of frequency dependence is not a general property of the barrier" as opposed to the Büttiker-Landauer [70] approach, which states that the maximum tunneling frequency $\omega$ is limited by the tunneling time $\tau$ through the relation $\omega \cdot \tau=1$. This means that tunneling at higher frequencies is still expected to happen with more efficiency than expected by the Büttiker-Landauer approach. Indeed, Liu's [71] experiment supports this finding where he states that "the tunneling time consideration is only an estimate."

In the end, it is worth noting that the recent models are less frequently used in the engineering field, and most of them are presented by physics research groups.

\subsection{Geometric Devices}

While most of the THz-rectifying diodes' behavior can be explained with quantum physics alone, geometric diodes seem to have comparably accurate simulation results, using the classical Drude model, to those obtained through quantum simulations without their complexity [72]. Geometric diodes are structurally different from other types and have some beneficial modeling features as will be explained.

These devices depend on physical asymmetry to simplify the movement of charge carriers into one direction more than the other. They are primarily ballistic devices that use long mean-free path length (MFPL) material to achieve this goal [64]. The recent development in the graphene industry and its properties has fostered its use for this goal such that a tapered graphene structure, as that shown in Figure 8, would facilitate the movement of electrons from the wider side to the narrower side. 
Technically, graphene devices have a very special feature compared to metals and semiconductors due to the linear energy dispersion relation near the Dirac point [73].

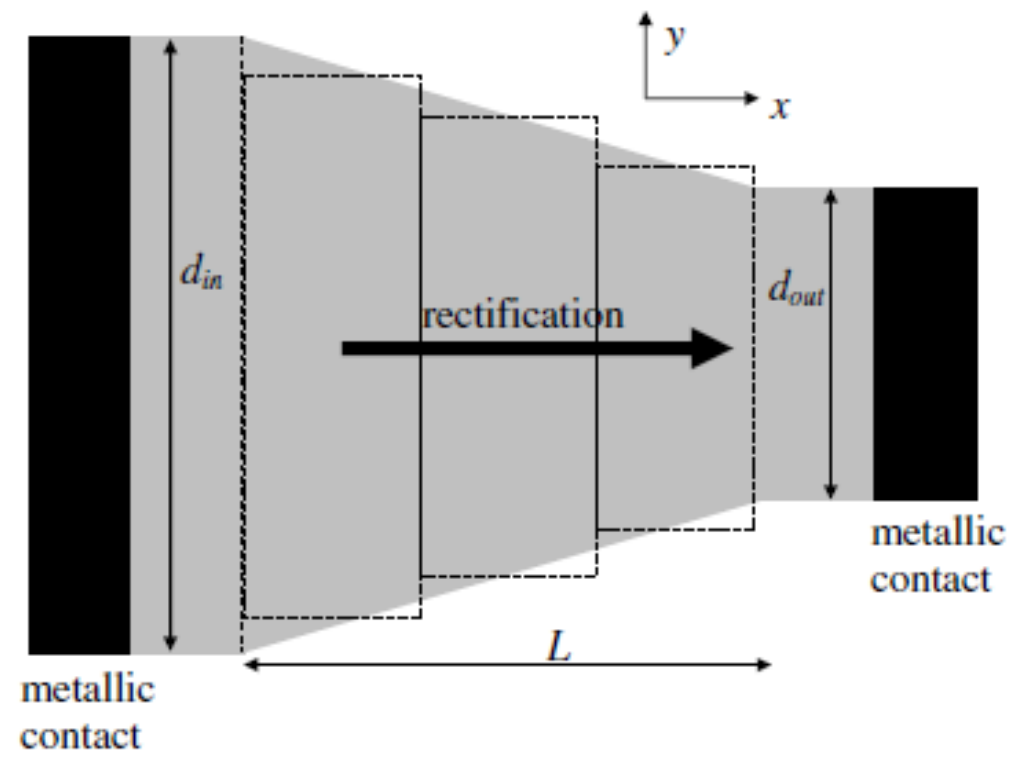

Figure 8. Schematic representation of the ballistic rectifier (Reproduced with permission from [74], IOP Publishing, 2013).

Modeling a device with this special energy and carrier transport features has also many approaches. However, there seems fewer theoretical frameworks and little agreement between experiment results and theoretical models' output. Therefore, it is worth reviewing some model types that witnessed many trials, whereas their outputs have been benchmarked against practical results. Dirac equation:

This equation was developed by Paul Dirac [75] based on the known wave equation to account for the relativistic particles. Dragoman and Dragoman [74] develop a model for the "2DEG (2DEG stands for two-dimensional electron gas) nanodevice with a linear tapered geometry working in the ballistic regime, where the transport of charge carriers is described by either the Schrödinger or the Dirac equation." Their model is based on Figure 8 where the device is sliced up into $\mathrm{N}$ cells such that the Dirac equation for each cell is given by (18).

$$
\begin{aligned}
\Psi_{j}(x, y) & =\sum_{n=1}^{N}\left[A_{n, j} \exp \left(i k_{n, j} x\right)\right. \\
& \left.+B_{n, j} \exp \left(-i k_{n, j} x\right)\right] \sin \left(2 n \pi y / d_{j}\right),
\end{aligned}
$$

In a similar style to other models, $A_{n, j}$ and $B_{n, j}$ are the incoming and outgoing wave components in slice $j ; k_{n, j}$ is the wave vector component and can be found by the knowledge of the potential $v$ and particle energy $E$. Working out some math and replacing the $j$ with the incident and outgoing indices in the following equation, the transmission probability $T$ for slice $j$ can be obtained from (19). Based on the knowledge of transmission probability, we can find the expected current value.

$$
T=\frac{\sum_{n=1}^{N_{\text {out }}} k_{n, \text { out }}\left|A_{n, \text { out }}\right|^{2}}{\sum_{n=1}^{N_{\text {in }}} k_{n, \text { in }}\left|A_{n, \text { in }}\right|^{2}},
$$

Although this method accounts for relativistic occurrences, the output results do not agree with measurements. This has been reported by Dragoman et al. [76] where they reported that the 
rectification region is less compared to that indicated by the simulation, and the measured current is a bit higher.

Monte Carlo simulation:

This simulation assumes electron movement and collision in geometric diode according to Drude model. In his work, Zhu [72] has developed a model where the electron collides with the structure boundaries, phonons, other electrons, etc., such that the electron moves within the device with velocity $v_{t o t}$, which is a composite of Fermi and current drift velocities. Stellingwerff [77] has also tried the Monte Carlo method for the simulation of his model.

Although there have been some important results from the work of Zhu [72], no agreement has been obtained between the modeled and measured current values. The work of Stellingwerff [77] does not deliver fundamentally different results where he argues that the simulation results provide the expectation of the measured results without directly comparing them. To sum up the current state-of-the-art for this algorithm, the output accuracy is disputed, and it can be used for knowledge of the general behavior of the device. Having said that, the simplicity of this method and avoidance of the more complicated quantum description is interesting.

It is worth noting that the NEGF method mentioned in Section 4.1 .1 can be used in the simulations of the geometric devices. However, there are few resources about the comparison between measured and modeled results for the $30 \mathrm{THz}$ region.

\subsection{Hybrid Diodes}

The reason we name them hybrid is because they have both features of geometric devices with large MFPL and junction diodes where a voltage drop is present at the junction between the graphene layer and the doped semiconductor substrate. They have earned recent interest based on their features [78]. The thermionic emission in conjunction with the Schottky-Mott model gives a surprisingly good description of their electrical properties as stated by Tongay, et al. [78].

Dragoman and Aldrigo have recently modeled this device and obtained efficiencies in excess of $58 \%$ at $897 \mathrm{GHz}$ for a graphene antenna on an n-doped GaAs substrate [79]. The Schottky-Mott model equation used in their study is (20).

$$
I(T, V)=A_{s u b}^{g r} \cdot A^{*} \cdot T^{2} \cdot \exp \left(-\frac{e \phi_{S B H}}{k_{B} T}\right) \cdot\left[\exp \left(\frac{e V}{\eta k_{B} T}\right)-1\right]
$$

where $I(T, V)$ is the current response of the device with respect to voltage and temperature, $A_{\text {sub }}^{g r}$ is the contact surface area between the graphene and the substrate, $A^{*}$ is the Richardson constant, $T$ is the temperature in Kelvin, $\phi_{S B H}$ is the Schottky barrier potential height, $V$ is the applied voltage value, $\eta$ is the ideality factor and $k_{B}$ is the Boltzmann constant.

Although the tried frequency is below the THz band, the modeling for this device results in exceptional efficiencies compared to all the experiments reviewed in this paper. The structure, also, benefits from the small capacitance of the graphene-substrate junction. However, no measured data are provided for this model to compare to in the $\mathrm{THz}$ band. It is also expected to obtain different responses at higher frequencies due to the increasing impact on the wave/Dirac function waveforms. Figure 9 shows the response of the modeled hybrid device compared to another MIM diode response. It shows that the response for the hybrid device is many orders of magnitude higher for lower available power levels. 


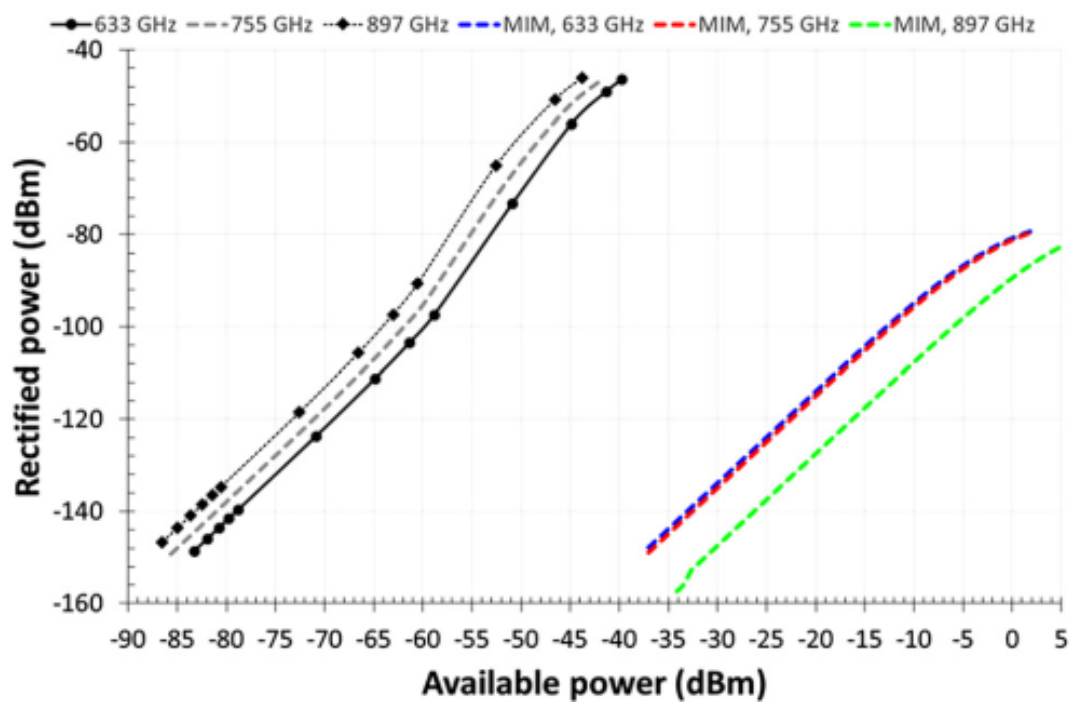

Figure 9. Available and rectified power levels for an MIM diode and a graphene Schottky junction diode. The colored graphs are for the MIM diode and the grey curves for the graphene Schottky junction case (Reproduced with permission from [79], AIP Publishing, 2016).

\section{Power Level}

The term power level is meant not only to refer to the amplitude of the incident wave, but also refers to its nature-narrowband or wideband - the spatial and phase correlation of the wave when it arrives at the receiving edge. Since the black body radiation law is deployed, the radiation, no matter at which frequency it occurs, has a wide band nature, is radiated by the whole surface it comes from and can be considered randomly polarized. Accounting for these factors will lead to a significant impact on the rectified wave efficiency, the best size for an antenna array to achieve optimum efficiency and of course the expected signal amplitude at the rectifier input. Mashaal and Gordon [34] have conducted a rigorous study to account for this impact for sunlight. Although this occurs at much higher frequencies, the results might be extended to lower frequencies as far as the electromagnetic wave theory is concerned. Therefore, the review of this factor is better approached through the issues addressed above as follows.

\subsection{Signal Amplitude}

Studies that address the efficient operation region of diodes for rectification are not new and may be dated back to the studies of Torry and Whitmer [80] where it was assumed to use a polynomial equation to describe the $I(V)$ relationship. The assumption of this nonlinear relationship leads to self-consistent equation forms in the simplest case. The recent development in the studies of rectenna diodes yields much more complicated relationships between voltage and current such as exponential, root power and nonlocal functions, just to name a few. While the nonlinearity behavior is considered of major importance, several recent papers overlook its importance and just focus on proving the nonlinearity under DC conditions. It is, therefore, necessary to deploy nonlinear solution schemes for circuits such as linearization [81] and harmonic balance [82]. An important feature of nonlinear systems is that any slight change in the working conditions can lead to unpredicted output, out of the operating range solution or even instability. Therefore, knowing the type of application the rectenna is assigned to and having a good estimate of the expected input power level are of extreme importance.

Based on the field of application and the expected operation power level, rectenna systems where the name rectenna is used interchangeably for both categories may be divided into: 
- Detectors: where it is important that the detector behavior is linear at least in the working range. It is worth noting that most of the recent work is dedicated to the use of the diodes as detectors $[45,72,83,84]$.

- Rectifiers: where it is of a major importance to express a good level of nonlinearity within the working range to increase the rectification efficiency for the harvested energy. In order to quantitatively characterize the diodes as efficient rectifiers, Donchev et al. [19] reinforced what has been set by $[50,85,86]$ as the criteria that a diode should satisfy to be considered a rectifier. The rectifier figures of merit are asymmetry $f_{a s y m}(V)$, nonlinearity $f_{N L}(V)$ and responsivity $f_{R E S}(V)$. They can be calculated using (21)-(23), respectively. In these equations, the current is represented as a function of voltage $I(V)$; forward and reverse current values for the same voltage absolute value are $I_{F}(V)$ and $I_{R}(V)$, respectively. The minimum figures of merit values required are shown next to the respective equations. Having said that, it should be noted that these devices are, after all, nonlinear where it is better to be approached subjectively. This means that these ruling figures are just for guidance, and each device design would have its own characteristics.

$$
\begin{aligned}
f_{\text {asym }}(V) & =\left|\frac{I_{F}(V)}{I_{R}(V)}\right| & f_{\text {asym }}(V)>1, \\
f_{N L}(V) & =\frac{d I(V)}{d V} / \frac{I(V)}{V} & f_{N L}(V)>3, \\
f_{R E S}(V) & =\frac{d^{2} I(V)}{d V^{2}} / \frac{d I(V)}{d V} & f_{\text {RES }}(V)>7 V^{-1},
\end{aligned}
$$

Having a good estimate of the power level is not only good to predict a stable mathematical solution, but also for physical stability!During the fabrication of the prototype, Zhu used in his work the geometric diode [72]; the prototype underwent what is referred to as "electromigration", which can tear down the structure under voltages as low as $0.1 \mathrm{~V}$.

Another important aspect regarding the power level is engineering the rectenna structure physically and geometrically (i.e., in terms of the good choice of material, doping concentration and the dimensions) in order to maximize the efficiency. To achieve this goal, it has been suggested to introduce asymmetry into the diode structure. While geometric diodes are by nature asymmetric, tunneling diodes, for example, can be made asymmetric by using different metals for the electrodes $[50,56,87]$.

Lastly, power level is closely connected to the frequency of operation. Therefore, the design should be aware of the both as shown in Section 3.

\subsection{Received Wave Bandwidth}

The black body radiation nature not only affects how the frequency components get rectified by the diode, but it also affects how the frequency components of the incident wave exchange energy between each other. In this context with reference to Figure 2, the bandwidth nature of the radiation would have a direct impact on $\eta_{a}$ and $\eta_{j}$ besides its known impact on matching. Therefore, the bandwidth will be prone to two impacts, which are given next.

\subsubsection{Impact from the Incident Wave}

A dedicated work is the one performed by Divit and Novotny [88] and by Mashaal and Gordon [34] where they conducted a study on solar light to identify the coherence of the incident ray in addition to the optimal antenna size to maximize the received power. It might be helpful to mention that Mashaal and Gordon utilize the equal time mutual coherence function (EMCF), which is identified in (24) based on the van Cittert-Zernike theorem.

$$
E M C F=\left\langle E(r, t) \cdot E^{*}\left(r^{\prime}, t\right)\right\rangle_{t}=2 \eta \int_{\sigma} I(s) \cdot \frac{e^{i k\left(r-r^{\prime}\right)}}{r \cdot r^{\prime}} d s
$$


According to the van Cittert-Zernike theorem, the time-averaged product of two fields emanating from a radiating source on two different points of an aperture antenna is given by (24) where: (i) $\eta$ is the vacuum impedance, (ii) $I(s)$ is the irradiance of the source, (iii) $r$ and $r^{\prime}$ are the coordinates of the different points of the aperture antenna and (iv) $k$ is the wave vector of the incident wave. The integration is performed over the entire radiating source surface $\sigma$. Mashaal and Gordon [34] have found significant results by running this integral for monochromatic and wideband sources in the visible range (For waste heat sources, the distance between the source and the rectenna array is very small. When integrating over the radiating surface, the aperture radius values that yield higher EMCF values will be considerably smaller than those obtained for the sunlight as in Mashaal and Gordon's study [34].). That is, the intercepted power by the antenna is proportional to the aperture area if the aperture radius is much smaller than the average wavelength $\bar{\lambda}$. However, when the aperture is much larger than the wavelength, the intercepted power by the antenna is independent of antenna size and relies only on the wavelength and the source irradiance. This sets limits how large the aperture should be. A more realistic approach might be reached by integrating the EMCF function over the entire bandwidth after introducing the frequency-dependent terms into (24). This has led to the EMCF curves shown in Figure 10, which shows that the situation is not much different for the wideband nature of light as compared to the quasi-monochromatic cases represented on the same graph.

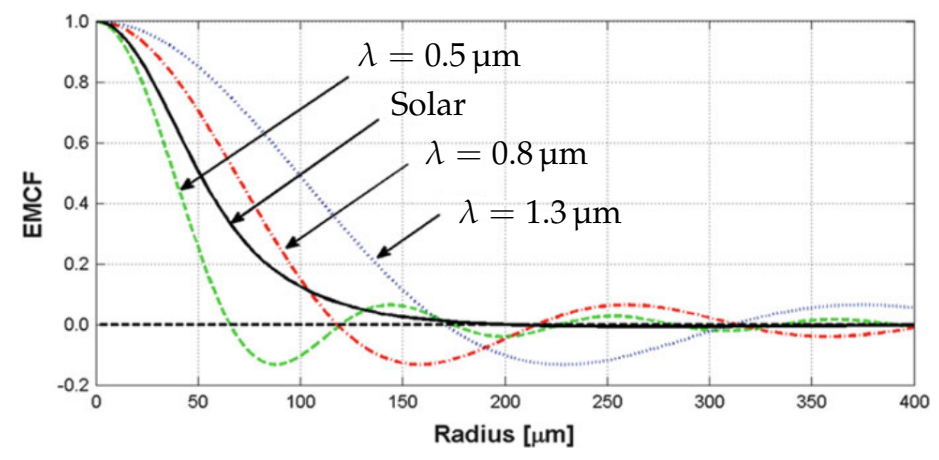

Figure 10. Equal time mutual coherence function (EMCF) function curve for the quasi-monochromatic light for wavelengths $\lambda=1.3 \mu \mathrm{m}, 0.9 \mu \mathrm{m}, 0.5 \mu \mathrm{m}$ and for solar black body radiation (Adapted with permission from [34], Springer, 2013).

From Figure 10, it can be concluded, except for the lowest wavelength $\lambda=0.5 \mu \mathrm{m}$, that for any aperture radius below $100 \mu \mathrm{m}$, quasi-monochromatic light is more likely to deliver higher power to this aperture than the complete bandwidth of the solar light. This result has been pronounced also by Divit and Novotny [88] suggesting that splitting the whole solar bandwidth into smaller sub-bands would yield higher efficiencies as the case for multi-junction solar cells. This solution can be extended to be applied also to rectennas, as this defect relies merely on the wideband nature of light and has nothing to do with the successor stages.

\subsubsection{Impact from the Rectifier Device}

This impact would quantitatively affect the $\eta_{j}$ as stated earlier. A good example might be attributed to Joshi and Moddel [66] where they studied the effect of the rectified wave bandwidth on the rectification efficiency starting from a monochromatic light, all the way to a wideband wave representing the black body radiation at sun temperature. The diode model is a simplified piecewise-linear diode model with zero reverse leakage current and was solved using the harmonic balance method. They found out that the efficiency can reach $100 \%$ for monochromatic light if the correct DC voltage is reached. However, the efficiency drops to $40 \%$ when a wideband signal is applied to the circuit. This number reminds us of the $44 \%$ Shockley-Queisser limit [9] calculated for p-n 
junction solar cells. Again, this study assumes a perfectly received wideband signal at the antenna side disregarding any of the aforementioned issues noted in the earlier section.

In fact, the findings for both impacts suggest that splitting the received wideband signal into narrower sub-bands will not only increase the wave coupling efficiency with the antenna $\eta_{a}$, but will also increase the diode efficiency $\eta_{j}$ as denoted in (1). In practice, thermophotovoltaics (TPV) have been around for many decades [89] and have recently become a hot research area [90-92]. Broadly speaking, they re-emit the absorbed radiation at different frequencies and bandwidths. DeMeo et al. [92] suggest using them with rectennas and propose many design prototypes. Some trials have taken place to commercialize their application $[89,93]$.

\subsection{Polarization}

Contrary to the widespread theoretical framework that deals with the electromagnetic radiation as a vectorized quantity, it is assumed that radiation of a black body is randomly polarized. Therefore, the formation of the EMCF function studied above is based on the assumption of unpolarized sources, which may hold true for solar radiation. At the same time, many recent experiments on rectennas show a polarization-dependent response. This has two major outputs: (a) this supports the hypothesis that their response is due to AC rectification, not a bolometric effect, as will be shown later, and (b) it can be said that the output assuming an unpolarized source will be different from that from a fully- or partially-polarized source [94]. The experiments in support of the polarization-dependent response of the rectenna are many, such as those of $[45,95,96]$. Bareiß et al. [96] have experimentally ensured the polarization-dependent response of the rectenna system reaching a polarization ratio of seven times in their experiment. Fumeaux et al. [28] have also concluded a polarization-dependent response of the rectenna system at $30 \mathrm{THz}$, but it should be noted that it was assumed to be composite, i.e., resulting from both tunneling and thermal effects.

\section{Thermal Effect}

Besides the several studies about the charge transition in rectenna system diodes, the thermal effect is always significant and present. This is due to the agitation of electrons in any temperature above absolute zero, $0 \mathrm{~K}$, which results in the thermionic activity [97]; another possible effect is the thermocouples, which can have many types as will be demonstrated. The thermal effects present in the diodes used in rectenna systems may be divided into three categories.

\subsection{Thermionic Effect}

This represents the effect on electrons when they are given enough energy by thermal agitation to leave the atom in the conduction band outside the metal. This effect has been studied by many researchers such as Becquerel, and Edison. It has been studied for the case of the tunneling diode by John Simmons [97] who found out that the current density value $J$ resulting from the thermionic emission when a voltage $V$ is applied to the diode is given by (25):

$$
J=A^{*} T^{2} e\left(\phi_{1}^{\prime} / k_{B} T\right)\left(1-e^{-e V / k_{B} T}\right),
$$

where $A^{*}$ is the Richardson constant, $T$ is the absolute temperature in $\mathrm{K}, \phi_{1}^{\prime}$ is the maximum value of the potential barrier with respect to the negative biased electrode, the left side barrier height, $K_{B}$ is Boltzmann constant and $V$ is the voltage applied to the diode. The combination of all these factors leads to a thermionic current density component. The same equation is used to calculate the thermionic current component in MIM diodes in [41,50], and so, it can be considered that the scientific basis to use this equation in tunneling diodes is well established. Certainly, there is another component, which is the tunneling component, whereas both components are present in the diode response. It is worth noting that geometric diodes will not have this type of thermal effect simply because there is no barrier traversal as in the tunneling diodes. 


\subsection{Seebeck Effect}

A recent trend in research considers another thermal effect that occurs between two different conductors, whereas the difference can either be in the nature of the conductor or even in their geometric shape as will be shown afterwards. This effect is referred to as the Seebeck effect, which has been stated by Goldsmid [98] as follows: "It is manifest as an electromotive force (EMF) or voltage $V$ which appears when the junction between two dissimilar conductors (A and B) is heated." In its simplest form, it may be represented by (26) as shown below.

$$
\nabla V=-S_{A B} \nabla T,
$$

where $\nabla T$ represents the temperature gradient in the studied conductor, $S_{A B}$ is the Seebeck coefficient difference between Conductors $\mathrm{A}$ and $\mathrm{B}$ and $\nabla V$ is the resulting potential difference gradient.

While the Seebeck effect was first introduced for junctions between different conductors, subsequent literature [99] experimentally shows that the Seebeck effect is dimension-dependent even for the same conductor. This fact has been deployed to obtain the Seebeck effect by fabricating thermocouples of the same conductor and introducing a discontinuity in the connection cross-section of the conductor [84]. Figure 11 represents the fabricated thermocouple rectenna with the metal junction discontinuity. However, the results of several experiments that have been conducted to find the Seebeck coefficient value based on measurements have led to contradicting results [27], which is a key challenge to reach an accurate model to calculate the efficiency of this type of thermocouple rectenna.

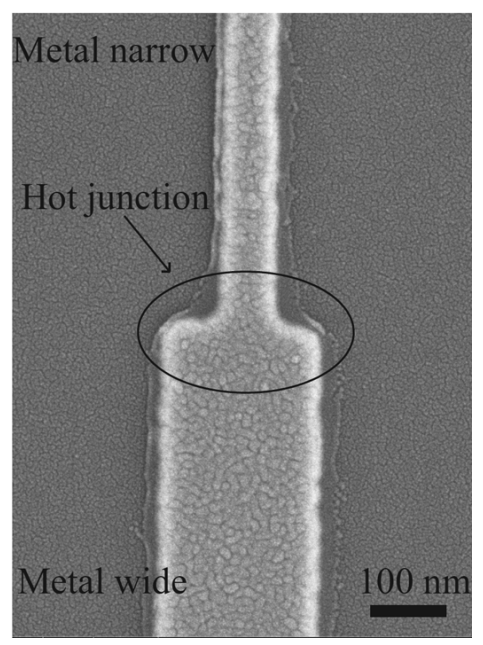

Figure 11. Fabricated thermocouple with a metal junction discontinuity (Reproduced with permission from [84], IEEE, 2015).

Therefore, it is expected to have the Seebeck effect for junctions between different conductors or even the same conductor. Here, disregarding the thermionic current component, an important question arises. That is: Is the output of the diodes used in rectennas attributed to the thermocouple effect or the charge particle transition mechanics demonstrated in earlier sections? In fact, experiments' output might be considered helpful to answer this question. The output of the experiment conducted by Bareiß et al. [96] addresses this issue such that the $I(V)$ characteristics of the fabricated diode could refer to the actual theory of operation. The fabrication process resulted in operating antennas (meaning having the expected output response) and defect antennas. In their experiment, they differentiate between them based on their $I(V)$ response. Through their work, they found out that the rectenna systems that showed the expected antenna response, where the rectenna output is polarization-dependent as a result of tunneling, had a relatively small resistance value in the order of kilo ohms. On the other hand, the rectennas that did not express a polarization-dependent response had a very high resistance values in the order of mega ohms. According to them, this can be attributed 
to the "resistive paths" formed through the insulator layer instead of having a tunneling behavior. This might suggest that a Seebeck effect is formed through these resistive paths in a similar fashion to that described by Russer et al. [84]. The high resistance property is also supported by the experiment of Li et al. [100] (This contradicts the low resistance requirement for tunneling-based diodes. For this reason, we have not discussed the dimensioning aspect. Instead, we focused on the ruling theories for these devices, which are critical to be identified before any dimensioning discussion is attempted.). There is a fine line between the polarization-dependent response of the antenna and the theory of operation. In other words, the previous description does not suggest that the Seebeck effect devices are polarization independent. The Seebeck thermal response might be due to the flowing current into the antenna or as a bolometric outcome when the substrate is heated by the incident light.

Primarily, Seebeck effect diodes are paired with detection applications as most of the reviewed literature reveals [101-103]. This is, for example, reinforced by Cuadrado et al. [103] who have experimented with two thermocouple antenna designs under two sources with different intensities. In their experiment, the response of their distributed bolometric effect rectenna system to a $10.6 \mu \mathrm{m}$ laser, with irradiance intensity of $1 \times 10^{8} \mathrm{~W} \mathrm{~m}^{-2}$, is $15 \mu \mathrm{V}$. Therefore, the focus is on the thermal antennas noise performance where the dominant parameter to quantify the performance is the noise equivalent power (NEP). It can be identified as "the value of the power $P$ that gives a unity signal-to-noise ratio for a bandwidth $\Delta v=1 \mathrm{~Hz}^{\prime \prime}$ as defined by Sanchez et al. [45]. In their model, they managed to reach NEP values around $100 \mathrm{pWHz}^{-1 / 2}$ for a barrier thickness around $10 \AA$ and frequency $10 \mathrm{GHz}$ with a fast roll off for THz frequencies and NEP values more than $5 \mathrm{nWHz}^{-1 / 2}$. Huhn et al. [101] for their uncooled BiSb/Sb thermocouple, experimentally managed to reach an NEP value below $200 \mathrm{pW} / \mathrm{Hz}^{-1 / 2}$ for a close-to-terahertz frequency of $812 \mathrm{GHz}$.

While it is quite normal for tunneling diode models to vary many orders of magnitude compared to experimental results, the Seebeck effect diode models usually express accuracy the same order of magnitude as their experimentally-tested prototypes, reaching $75 \%$ accuracy as the case of Cuadrado et al. [103]. The reported results by Huhn et al. [101] show also good agreement between measured and modeled results.

\subsection{Other Thermal Effects}

In some cases, the device shows both tunneling and thermal behavior, where the polarization-dependent amplitude $V_{p}$ of the rectenna response is comparable to the polarization independent $V_{i p}$ response of the rectenna system. Figure 12 shows the polarization-dependent DC response of a $\mathrm{Ni}-\mathrm{NiO}-\mathrm{Ni}$ symmetric tunneling diode rectenna with the contributions of both voltages. It is worth noting that the response shown in Figure 12 remains the same for the different contact areas used in this experiment. This behavior may be attributed to thermal-assisted tunneling ([46], p. 161) as the writer indirectly suggests.

In the end, the thermal effect might reveal itself in many forms and even have different reasoning. While the works of $[84,96,101,103]$ rely on the Seebeck effect to explain their results and obtain accurate models, Fumeaux et al. [28] and Heiblum et al. [46] attribute the output of their experiments to thermal-assisted tunneling. It should be noted, however, that Heiblum et al. [46] state in their paper that "lower impedance junctions had less thermal contributions than the high impedance ones"; which holds true for the experiment results of Seebeck effect model even with a different cause. Therefore, it can be empirically said that with an increased resistance value of the diode, higher thermal contribution is expected. 


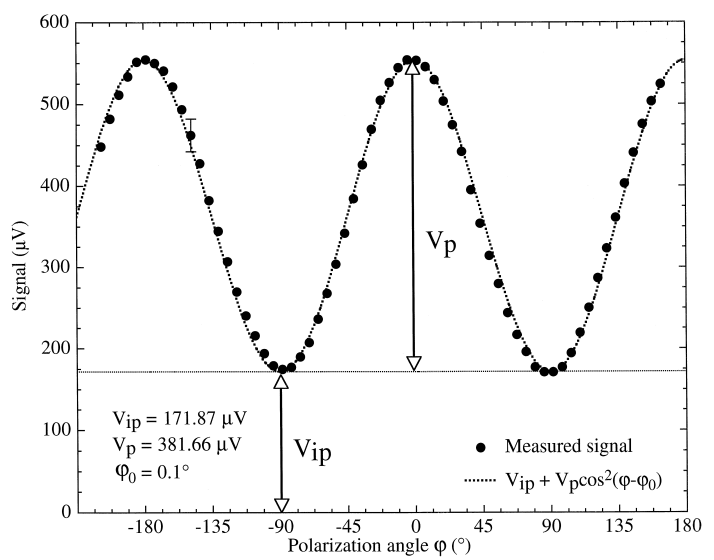

Figure 12. Polarization-dependent and independent response of the tunneling diode with the values of both components shown in the figure. Diodes are connected to the integrated dipole and bow tie antennas as described in [28]. The linear polarization angle $\phi$ of the incident radiation is changed, and the DC response of the diode is monitored. A $10.6 \mu \mathrm{m}$ laser was used with other facilities to obtain $5 \times 10^{6} \mathrm{~W} \mathrm{~m}^{-2}$ intensity at the antenna under test (Reproduced with permission from [28], Elsevier, 1998).

\section{Fabrication Aspects}

Another important factor when deciding the optimum rectifier design is how the design will be realized. Realization of the rectifier should address: (a) a reliable production process and (b) comparable or lesser costs than other commercially available solutions. In fact, there are many production methods where each has its own advantages and drawbacks. Since the technology is highly dynamic, suffers from synonymy (meaning that the same production procedure can be named differently by different institutions) and can significantly change within a short time, our discussion will not focus on the detailed process of each fabrication method. Rather, we will focus on the general concepts of the methods used for experiments, as well as those expected to be deployed for commercial production.

Producing nano-scale components with satisfactory accuracy has been a challenge. There are many examples where the produced prototypes fail to produce the required functionality due to fabrication deficiencies. Berland [5] stressed the importance of MIM diodes' reproducibility as the main step to increasing the efficiency of the rectennas. It has been demonstrated in Section 6 with the novel fabrication method used by Bareiß et al. [96] that some MIM diodes did not express a tunneling behavior as a consequence of fabrication flaws. Another interesting example is that of Conley and Alimardini ([37], p. 119) where extremely rough surface metal-insulator contacts can have significant impact on the diode response. The formation of the insulating layer in a tunneling diode is also not an exception where the crystallization form of the layer significantly affects its dielectric constant [42]. Therefore, reaching an accurate procedure to fabricate rectenna devices was the primary focus of many researchers.

In fact, there are many methods used today to fabricate nano-scale features ranging from current lithographic techniques to mechanical nano-imprint and other advanced techniques that involve transferring the imprinted structure into a foreign or flexible substrate [104]. These methods can be differentiated based on different chemical agents, fabrication procedure, exposure time, etc. We, therefore, list some of the most frequently-used methods as they appear in the literature.

\subsection{Current Lithographic Techniques}

The key difficulty to fabricate nano-scale features with high definition lies in the way they are produced. Out of many available lithographic techniques, short wavelength photo-masking has been suggested and is referred to as extreme ultra violet lithography (EUVL) [105]. However, it is 
hampered by many difficulties relevant to cleaning and repairing [106]. Furthermore, the decreasing feature dimension would lead to increased fabrication costs due to the technological difficulty [107]. Moreover, these technologies are primarily concerned with fabricating the features on silicon, GaAs, etc., substrates, which are the ones used for commercial solar cells. Therefore, the cost is certainly higher just because of using the same substrate. Regardless of the technical details, it is expected that individual etching or writing of the nano features would be more expensive than mass stamping them. This is the motivation for the next fabrication method.

\subsection{Nano Imprint Lithography}

Referred to in the literature as nano imprint lithography (NIL), this type joins the mass production benefit of using photomask lithography and the high-resolution lithography techniques-laser, e-beam, etc.-which are known for their high resolution [107].

The following briefly describes the generic fabrication method that can be used to produce nano-scale circuits or features on a substrate. The feature is fabricated in the typical way, which can incorporate many depositing and etching stages either in additive mode where the required material is deposited over the photoresist and then removing the photoresist and leaving the remaining material as the required layer; or in subtractive mode, where the material is deposited before the photoresist, which leaves the unwanted material exposed. This exposed material is etched away by plasma etching, for example, and the wanted layer will be under the photoresist layer, which can be lifted off at another stage, leaving the required material layer print. The photoresist mask itself will be fabricated based on the classical ways such as spin coating for depositing the photoresist layer. Additionally, ultraviolet, laser beam, e-beam, etc., or even another photomask above it and etching the unwanted photoresist areas by a photo-based process for etching of the photo-mask for additive and/or subtractive photo-masking can be used. The known techniques such as chemical vapor deposition (CVD), atomic layer deposition (ALD), etc., are used for depositing the material layer. Of course, the sequence of etching and depositing is relevant to the type, be it additive or subtractive processing. If further information is needed, the reader is advised to review [107] (Figure 16.1-3).

While this procedure takes a screening style through the use of photo-masking, another very high resolution direct writing procedure is available. It is known, however, for the very long production time because the feature has to be produced in a linear writing style.

\subsection{Mechanical Nano Imprint Lithography}

This type extends the aforementioned type where the photoresist is mechanically printed instead of being etched. The printing mold can be from any material and can be produced through the previous process. Slafer [107] refers to this mold as the stamp or the master template. The photoresist layer is deposited on the required substrate, and the stamp is applied to it, forming the required pattern, as shown in Figure 13.
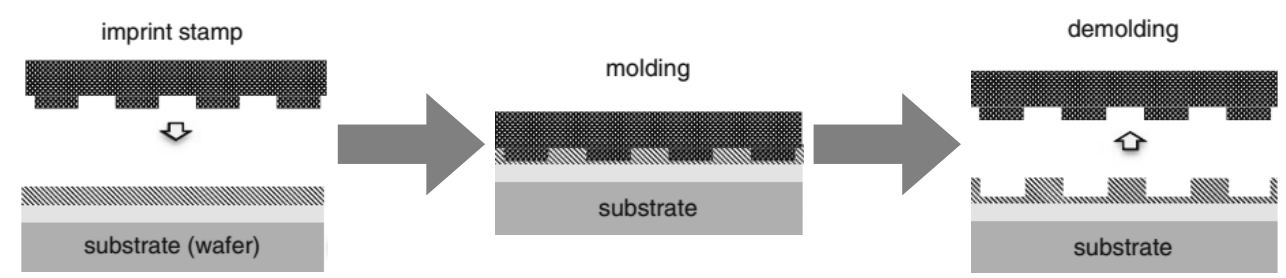

Figure 13. Nano imprint lithographic stamp applied to a polymer layer. After polymer solidification, a template that is equivalent to that of a photoresist mask is formed as shown in the final step after demolding (Adapted with permission from [107], Springer, 2013).

Now that the stamp has been formed, the polymeric resist masks are stamped onto the material to continue the fabrication process. There are several ways to develop the imprinted photoresist based 
on its type, i.e., solid or liquid. The stamps can be made from metal, quartz, etc. However, the stamps produced by writing facilities such as laser, e-beam, etc., take very long to finish due to the linear writing type and are as expensive as $\$ 10,000$ for a few square centimeters [107]. One good substitution for mass production of rectenna solar cells is the polymer stamps, which cost less than $\$ 1000$ to produce the same photoresist stamp [107].

\subsection{Roll to Roll Manufacturing}

While the previous type of production of nano printed features slightly helps reduce the price and offers production with larger capacities, roll to roll manufacturing (R2R) can be a promising technology to produce rectennas at much lower prices. In fact, the lump cost comes from using the substrate, be it silicon or any other material. Slafer [107] argues that producing nano features on foldable structures can be even less expensive. This argument has good background as the cost of producing hologram-printed features that are used on credit cards and authentic trade marks is around $\$ 0.15$ per square meter. In fact, using foldable rectenna array structures to harvest waste heat energy can offer more flexibility.

Slafer ([107] (Figure 16.15)) suggests the following solution, shown in Figure 14, to mass-produce rectennas at reduced prices. In a recent follow up [108], the patented work is for addressing many fabrication issues by using inexpensive multilayer plastic printing drums to help deliver light to the photoresist printed layer through the roll to roll fabrication process. Moreover, the patent suggests using "solvent/diluent" mixture to use in the fabrication process of thermally-developed polymer photoresists. It is advantageous because it does not require the elevated temperatures that may damage other temperature-sensitive features already printed, as suggested by the author.

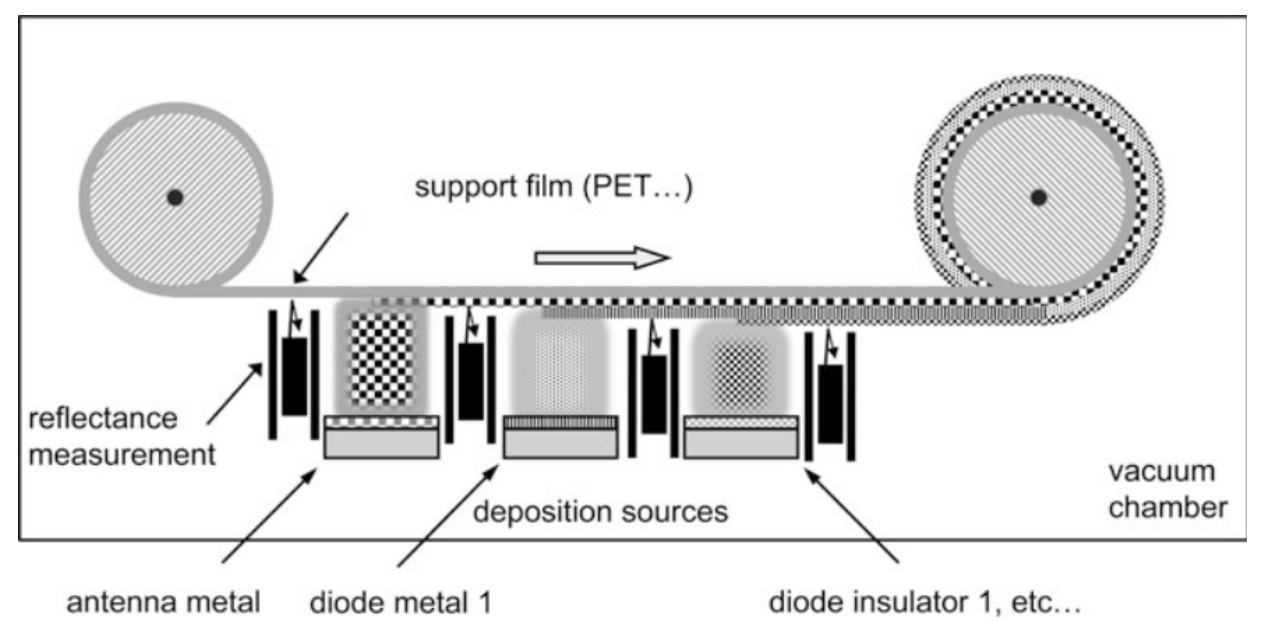

Figure 14. The suggested roll to roll chamber to produce rectennas (Reproduced with permission from [107], Springer, 2013).

\subsection{Nano Transfer Print}

Nano transfer print (nTP) is also another fabrication process that incorporates printing as NIL, but is different in two aspects: (a) it is suggested for printing on a substrate layer; and (b) the whole feature is printed; meaning that the whole diode is printed as shown in Figure 15. In the stack of Figure 15d, the first deposited layer is the organic self-assembled monolayer (SAM) to provide weak adhesion to the stamp surface; this is followed by AuPd alloy that is weakly attached to the SAM surface; the aluminum deposition follows this as the first metal in the actual MIM diode; the insulating layer is then deposited followed by another AuPd alloy layer; and finally, an adhesion promoter, which can be titanium or chrome. The gold palladium alloy has been used because it provides surface roughness of less than $1 \mathrm{~nm}$ [109], which is already known for its impact on the predictability of the diode functionality [37]. 
a

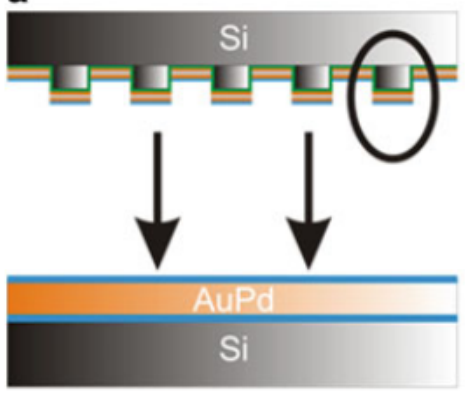

C

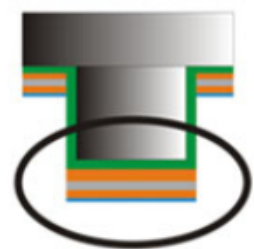

b

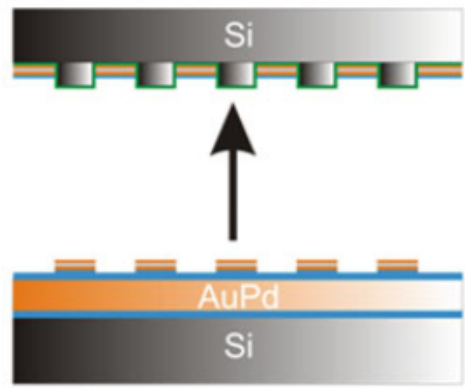

d

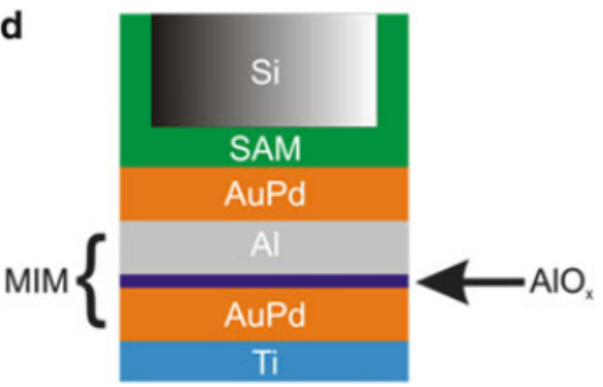

Figure 15. The nano transfer printing method. (a) shows how the stamp looks before the printing where diodes are still on the stamp protrusions; (b) since the organic self-assembled monolayer (SAM) in the figures provides weak adhesion to the stamp surface, the diodes are transferred to the substrate when the stamp is applied to the substrate; the diode stack between the stamps remains there without getting printed because it has no contact with the substrate surface; (c) close-up view of the stamp; (d) shows the diode stack up reaching the stamp protrusion (Reproduced with permission from [109], Springer, 2013).

The same group [96] managed to use the same method to fabricate the diode integrated with an antenna network at the nano-scale. It should be noted, however, that there have been flaws in the fabrication process, which resulted in sections that did not follow the tunneling rule. Little information is available in that paper about how many sectors have the unpredicted response. Weiler et al. [110], on the other hand, managed to reliably fabricate metal oxide substrate (MOS) junctions of $\mathrm{Au} / \mathrm{Ti} / \mathrm{TiO}_{x} / \mathrm{p}^{+}$-Si measuring below $70 \mathrm{~nm}$. Furthermore, Nagel et al. [111] have addressed the use of this method in the fabrication of circuits measuring below $50 \mathrm{~nm}$ using a stamp upon which the rectennas can be deposited. Their method looks similar to that of Slafer discussed above in the sense of reproducing the stamp from the master template. The difference is that here, the whole device is deposited before stamping. They, quantitatively, evaluate the performance of the method where over $70 \%$ of the produced devices are operative if the printing time is about $40 \mathrm{~min}$ under set conditions as shown in ([111], Figure 4d).

In the end, the nTP method provides a deep understanding of the adhesion of stacked materials, but its literature is still based on the printing on silicon or MOS substrates where the biggest cost for device fabrication is concentrated. Another practical aspect also refers to the fabrication of extremely tiny nanometer structures such as that described by Mayer et al. [36], who propose a metal-vacuum-metal tunneling diode structure able to rectify wavelengths up to green light with high efficiency!The structure relies on a metal tip protruding in the vacuum opposite a flat anode. The fabrication of such a device with the available technology is a big challenge, although there are ongoing trials on this matter at an experimental scale. 


\section{Conclusions}

In this paper, some recent and earlier work on rectennas and their applications has been reviewed. More focus has been given to the factors that contribute to the decision-making design process, giving particular attention to the frequency, theory of operation and specific types of diodes.

While sunlight provides an extremely high radiation intensity, we think it is unlikely to obtain an efficient design for frequencies in the several hundred $\mathrm{THz}$ range with the available technology for today. Lower frequency resources referred to as waste heat sources that radiate in the $30 \mathrm{THz}$ range offer good abundance and have more encouraging experimental results, although their power is limited.

While many forms of $\mathrm{THz}$ rectennas having been suggested, designed and even fabricated, there is no common ground among researchers on the best design or even the theory of operation. As some researchers assume tunneling as the dominant mechanism for their operation, others rely on the ballistic effect to build their models. Be it this or that, there is also the thermal effect, which is always present in more than one form. In order to simplify the election process, relying on the figures of merit given by (21)-(23) would yield an easier and clearer decision-making process. Therefore, out of the many available designs, tunneling, geometric and hybrid diodes hold high potentiality for deployment for this frequency range in any future design because their figures of merit are close to those imposed by Equations (21)-(23), and reaching the required figures is a matter of optimizing the diode design.

Having said that, the general trend in the recent research overlooks the importance of matching between the diode and the antenna and just focuses on the proof of concept for the physical phenomenon, be it tunneling or ballistic carrier transportation. On the other hand, these diodes' $I(V)$ relationship is not only nonlinear, but it can also be nonlocal with respect to time, so imposing more importance on accounting for this behavior in any future simulation. In other words, to optimize the models to reach efficient designs, the future model must take into account the physical aspect from the perspective of charge transport and the electrical aspect from the perspective of dynamic signal models together to have a more accurate and realistic simulation.

Doing so is not easy, however, because the carrier transport is often subject to quantum physics at high frequencies. Especially, as the wave (Schrödinger) equation is used to describe the carrier transport at the mesoscopic scale, it incorporates many parameters when solving a quantum problem. Therefore, the modeled systems are not only quantumly complicated, but there is also a subjectivity issue. In other words, each device would need a separate physical model to describe its operation. We support this view, for example, with the models of Simmons and Sanchez et al., who tried to formulate one model linking physical and electrical parameters in a few equations. Further tests on the models of Simmons and Sanchez et al. approved that they are neither expandable nor amendable to account for higher frequencies as they have structural limitations in their formalism. On the other hand, having a separate simulation for each build of a system would be both computer and resource intensive and can be an intimidating work to do for many researchers. Therefore, we think that for any future and efficient development of the rectenna device, it is of primary importance to reach an accuracy-cost-compromising model such that acceptably accurate results can be reached without imposing too complicated physical models.

Another culprit that can also distort the experimental output of a rectenna system is the presence of thermal effects. While the thermionic effect is expected to be present in many diode designs, Seebeck and thermally-assisted tunneling effects can be present, as well. In this scenario, we advise to design diodes with as small resistance values as possible based on the empirical findings by the experiments reviewed. This issue can also be addressed in any future research endeavor because of its importance for the accurate estimation of the device response.

Using this application for any future design is deeply connected with reducing its production costs. Today, on the other hand, all of the research in this field is merely performed on a solid substrate such as silicon or GaAs, which impose the largest cost of the device. Future and commercial implementations necessitate using much less expensive alternatives where we think that production technologies such 
as R2R or nTP hold good potentiality, as they might be used to print the device on flexible and less expensive surfaces. This can offer a very friendly solution suitable for use with curved waste heat radiating surfaces.

Author Contributions: These authors have contributed equally to this work

Conflicts of Interest: The authors declare no conflict of interest.

\section{References}

1. Landis, F.; Schenker, O.; Tovar Reaños, M.; Vonnahme, C.; Zitzelsberger, S. An Overview on Current Climate Policies in the European Union and its Member States; ENTRACTE Project Report; ENTRACTE: Washington, DC, USA, 2013.

2. WWF. How Low? Achieving Optimal Carbon Savings from the UK's Existing Housing Stock; WWF: Grand, Switzerland, 2008.

3. Corkish, R.; Green, M.; Puzzer, T. Solar energy collection by antennas. Sol. Energy 2002, 73, 395-401.

4. Brown, W.C. Optimization of the Efficiency and Other Properties of the Rectenna Element. In Proceedings of the IEEE-MTT-S International Microwave Symposium, Cherry Hill, NJ, USA, 14-16 June 1976; pp. 142-144.

5. Berland, B. Photovoltaic Technologies Beyond the Horizon: Optical Rectenna Solar Cell, Final Report, 1 August 2001-30 September 2002; Technical Report; National Renewable Energy Lab.: Golden, CO, USA, 2003.

6. Edmond Becquerel. Available online: https://en.wikipedia.org/w/index.php?title=Edmond_Becquerel\& oldid=791838776 (accessed on 7 August 2017).

7. Anonymous April 25, 1954: Bell Labs Demonstrates the First Practical Silicon Solar Cell. APS Phys. 2009, 18.

8. Li, Y. Synthesis, Characterization, and Photovoltaic Applications of Mesoscopic Phthalocyanine Structures. Ph.D. Thesis, Electrical Engineering and Computer Science Department, South Dakota State University, Brookings, SD, USA, 2011.

9. Shockley, W.; Queisser, H.J. Detailed balance limit of efficiency of p-n junction solar cells. J. Appl. Phys. 1961, $32,510-519$.

10. Sohrabi, F.; Nikniazi, A.; Movla, H. Optimization of Third Generation Nanostructured Silicon- Based Solar Cells. In Solar Cells—Research and Application Perspectives; Morales-Acevedo, A., Ed.; InTech: Rijeka, Croatia, 2013; Chapter 01.

11. Kazmerski, L.; Gwinner, D.; Hicks, A. Best research cell efficiencies. Natl. Renew. Energy Lab. 2010, 2.

12. King, R.; Law, D.; Edmondson, K.; Fetzer, C.; Kinsey, G.; Yoon, H.; Sherif, R.; Karam, N. 40\% efficient metamorphic GaInP/GaInAs/Ge multijunction solar cells. Appl. Phys. Lett. 2007, 90, 183516.

13. Michael, G.; Alan, G. A Manufacturing Cost Analysis Relevant to Single- and Dual-Junction Photovoltaic Cells Fabricated with III-Vs and III-Vs Grown on Czochralski Silicon; Report; NREL: Washington, DC, USA, 2013.

14. Yang, Y.; You, J. Make perovskite solar cells stable. Nature 2017, 544, 155-156.

15. Brown, W.C. The Microwave Powered Helicopter. J. Microw. Power 1966, 1, 1-20.

16. Moddel, G.; Grover, S. Rectenna Solar Cells; Moddel, G., Grover, S., Ed.; Springer: New York, NY, USA, 2013; 399p.

17. Koert, P.; Cha, J.; Machina, M. 35 and $94 \mathrm{GHz}$ rectifying antenna systems. In SPS 91-Power from Space; Société des Electriciens et des Electroniciens: Paris, France, 1991; pp. 541-547.

18. Chiou, H.K.; Chen, I.S. High-Efficiency Dual-Band On-Chip Rectenna for 35-and 94-GHz Wireless Power Transmission in 0.13-m CMOS Technology. IEEE Trans. Microw. Theory Tech. 2010, 58, 3598-3606.

19. Donchev, E.; Pang, J.S.; Gammon, P.M.; Centeno, A.; Xie, F.; Petrov, P.K.; Breeze, J.D.; Ryan, M.P.; Riley, D.J.; Alford, N.M. The rectenna device: From theory to practice (a review). MRS Energy Sustain. 2014, 1, doi:10.1557/mre.2014.6.

20. Moddel, G. Will rectenna solar cells be practical? In Rectenna Solar Cells; Springer: Berlin, Germany, 2013; pp. 3-24.

21. Konopsky, V.N.; Alieva, E.V. Long-range plasmons in lossy metal films on photonic crystal surfaces. Opt. Lett. 2009, 34, 479-481.

22. Optical Rectenna. Available online: https://en.wikipedia.org/w/index.php?title=Optical_rectenna\&oldid= 791785571 (accessed on 11 August 2017). 
23. Iluz, Z.; Boag, A. Dual-Vivaldi wideband nanoantenna with high radiation efficiency over the infrared frequency band. Opt. Lett. 2011, 36, 2773-2775.

24. Sabaawi, A.M.; Tsimenidis, C.C.; Sharif, B.S. Overview of nanoantennas for solar rectennas. In Rectenna Solar Cells; Springer: Berlin, Germany, 2013; pp. 231-256.

25. Masotti, D.; Costanzo, A.; Rusticelli, S.; Tartarini, G.; Aldrigo, M. Infrared nano-rectennas exploiting on-demand laser sources. In Proceedings of the 2014 IEEE RFID Technology and Applications Conference (RFID-TA), Tampere, Finland, 8-9 September 2014; pp. 17-20.

26. Grover, S.; Moddel, G. Optical frequency rectification. In Rectenna Solar Cells; Springer: Berlin, Germany, 2013; pp. 25-46.

27. Russer, J.A.; Jirauschek, C.; Szakmany, G.P.; Schmidt, M.; Orlov, A.O.; Bernstein, G.H.; Porod, W.; Lugli, P.; Russer, P. High-Speed Antenna-Coupled Terahertz Thermocouple Detectors and Mixers. IEEE Trans. Microw. Theory Tech. 2015, 63, 4236-4246.

28. Fumeaux, C.; Herrmann, W.; Kneubühl, F.K.; Rothuizen, H. Nanometer thin-film Ni-NiO-Ni diodes for detection and mixing of $30 \mathrm{THz}$ radiation. Infrared Phys. Technol. 1998, 39, 123-183.

29. Masotti, D.; Costanzo, A.; Aldrigo, M.; Dragoman, M. Graphene-based nano-rectennain the far infrared frequency band. In Proceedings of the 2014 44th European Microwave Conference, Rome, Italy, 6-9 October 2014.

30. Dragoman, M.; Aldrigo, M.; Dinescu, A.; Dragoman, D.; Costanzo, A. Towards a terahertz direct receiver based on graphene up to $10 \mathrm{THz}$. J. Appl. Phys. 2014, 115, doi:10.1063/1.4863305.

31. Vakil, A.; Bajwa, H. Energy harvesting using Graphene based antenna for UV spectrum. In Proceedings of the 2014 IEEE Long Island Systems, Applications and Technology Conference (LISAT), Farmingdale, NY, USA, 2 May 2014; pp. 1-4.

32. Byrnes, S.J.; Blanchard, R.; Capasso, F. Harvesting renewable energy from Earth's mid-infrared emissions. Proc. Natl. Acad. Sci. USA 2014, 111, 3927-3932.

33. Kopp, G.; Lean, J.L. A new, lower value of total solar irradiance: Evidence and climate significance. Geophys. Res. Lett. 2011, 38, doi:10.1029/2010GL045777.

34. Mashaal, H.; Gordon, J.M. Solar and thermal aperture antenna coherence performance limits. In Rectenna Solar Cells; Springer: Berlin, Germany, 2013; pp. 69-86.

35. Thermionic Emission. Available online: https://en.wikipedia.org/w/index.php?title=Thermionic emission\&oldid=789623260 (accessed on 14 August 2017).

36. Mayer, A.; Chung, M.; Weiss, B.; Miskovsky, N.; Cutler, P. Three-dimensional analysis of the rectifying properties of geometrically asymmetric metal-vacuum-metal junctions treated as an oscillating barrier. Phys. Rev. B 2008, 78, 205404.

37. Conley, J.F., Jr.; Alimardani, N. Impact of Electrode Roughness on Metal-Insulator-Metal (MIM) Diodes and Step Tunneling in Nanolaminate Tunnel Barrier Metal-Insulator-Insulator-Metal (MIIM) Diodes. In Rectenna Solar Cells; Springer: Berlin, Germany, 2013; pp. 111-134.

38. HyperPhysics. Wien's Displacement Law. 2006. Available online: http://hyperphysics.phy-astr.gsu.edu/ hbase/wien.html (accessed on 14 August 2017).

39. Tucker, J.R.; Feldman, M.J. Quantum detection at millimeter wavelengths. Rev. Modern Phys. 1985, $57,1055$.

40. Habbal, F.; Danchi, W.C.; Tinkham, M. Photon-assisted tunneling at 246 and $604 \mathrm{GHz}$ in small-area superconducting tunnel junctions. Appl. Phys. Lett. 1983, 42, 296-298, doi:10.1063/1.93885.

41. O'Regan, T.; Chin, M.; Tan, C.; Birdwell, A. Modeling, Fabrication, and Electrical Testing of Metal-Insulator-Metal Diode; Technical Report; Army Research Lab Adelphi Md Sensors and Electron Devices Directorate: Adelphi, MD, USA, 2011.

42. Hathaikarn, M. Electrical Properties of Niobium Based Oxides-Ceramics and Single Crystal Fibers Grown by the Laser-Heated Pedestal Growth (LHPG) Technique. Ph.D. Thesis, The Graduate School Intercollege Graduate Program in Materials, The Pennsylvania State University, Centre County, PA, USA, 2003; p. 319.

43. Polyanskiy, M.N. Available online: https:// refractiveindex.info (accessed on 30 August 2017).

44. Simmons, J.G. Generalized formula for the electric tunnel effect between similar electrodes separated by a thin insulating film. J. Appl. Phys. 1963, 34, 1793-1803.

45. Sanchez, A.; Davis, C., Jr.; Liu, K.; Javan, A. The MOM tunneling diode: Theoretical estimate of its performance at microwave and infrared frequencies. J. Appl. Phys. 1978, 49, 5270-5277. 
46. Heiblum, M.; Wang, S.; Whinnery, J.; Gustafson, T. Characteristics of integrated MOM junctions at dc and at optical frequencies. IEEE J. Quantum Electron. 1978, 14, 159-169.

47. Grover, S.; Moddel, G. Engineering the current-voltage characteristics of metal-insulator-metal diodes using double-insulator tunnel barriers. Solid-State Electron. 2012, 67, 94-99.

48. Smythe, W.R. Static and Dynamic Electricity; McGraw-Hill Book Company, Inc.: New York, NY, USA, 1950.

49. Miller, S.C., Jr.; Good, R., Jr. A WKB-type approximation to the Schrödinger equation. Phys. Rev. 1953, 91,174 .

50. Chin, M.L.; Periasamy, P.; O’Regan, T.P.; Amani, M.; Tan, C.; O’Hayre, R.P.; Berry, J.J.; Osgood, R.M., III; Parilla, P.A.; Ginley, D.S. Planar metal-insulator-metal diodes based on the $\mathrm{Nb} / \mathrm{Nb} 2 \mathrm{O} 5 / \mathrm{X}$ material system. J. Vac. Sci. Technol. B Nanotechnol. Microelectron. Mater. Process. Meas. Phenom. 2013, 31, doi:10.1116/1.4818313.

51. Koppinen, P. Bias and Temperature Dependence Analysis of the Tunneling Current of Normal Metal-Insulator-Normal Metal Tunnel Junctions. Master's Thesis, Departmment of Physics, University of Jyväskylä, Jyväskylä, Finland, 2003.

52. Transfer-Matrix Method (Optics). Available online: https:/ / en.wikipedia.org/w /index.php?title=Transfermatrix_method_(optics)\&oldid=792683168 (accessed on 17 August 2017).

53. Jonsson, B.; Eng, S.T. Solving the Schrodinger equation in arbitrary quantum-well potential profiles using the transfer matrix method. IEEE J. Quantum Electron. 1990, 26, 2025-2035.

54. Bastard, G. Superlattice band structure in the envelope-function approximation. Phys. Rev. B 1981, $24,5693$.

55. Jirauschek, C. Accuracy of transfer matrix approaches for solving the effective mass Schrödinger equation. IEEE J. Quantum Electron. 2009, 45, 1059-1067.

56. Hashem, I.E.; Rafat, N.H.; Soliman, E.A. Theoretical study of metal-insulator-metal tunneling diode figures of merit. IEEE J. Quantum Electron. 2013, 49, 72-79.

57. Burt, M. Fundamentals of envelope function theory for electronic states and photonic modes in nanostructures. J. Phys. Condens. Matter 1999, 11, 53.

58. Lent, C.S.; Kirkner, D.J. The quantum transmitting boundary method. J. Appl. Phys. 1990, 67, 6353-6359, doi:10.1063/1.345156.

59. Filipovic, L.; Stanojevic, Z.; Baumgartner, O.; Kosina, H. 3D modeling of direct band-to-band tunneling in nanowire TFETs. Energy 2014, 1, 1-5.

60. Paulsson, M. Non equilibrium green's functions for dummies: Introduction to the one particle NEGF equations. arXiv 2002, arXiv:cond-mat/0210519.

61. Perturbation Theory (Quantum Mechanics). Available online: https://en.wikipedia.org/w/index.php? title=Perturbation_theory_(quantum_mechanics)\&oldid=793439215 (accessed on 19 August 2017).

62. Grover, S.; Moddel, G. Metal single-insulator and multi-insulator diodes for rectenna solar cells. In Rectenna Solar Cells; Springer: Berlin, Germany, 2013; pp. 89-109.

63. Datta, S.; Anantram, M. Steady-state transport in mesoscopic systems illuminated by alternating fields. Phys. Rev. B 1992, 45, 13761.

64. Zhu, Z.; Joshi, S.; Grover, S.; Moddel, G. Geometric diodes for optical rectennas. In Rectenna Solar Cells; Springer: Berlin, Germany, 2013; pp. 209-227.

65. Tien, P.; Gordon, J. Multiphoton process observed in the interaction of microwave fields with the tunneling between superconductor films. Phys. Rev. 1963, 129, 647.

66. Joshi, S.; Moddel, G. Efficiency limits of rectenna solar cells: Theory of broadband photon-assisted tunneling. Appl. Phys. Lett. 2013, 102, 083901.

67. Floquet Theory. Available online: https://en.wikipedia.org/w/index.php?title=Floquet_theory\&oldid= 790708503 (accessed on 21 August 2017).

68. Grifoni, M.; Hänggi, P. Driven quantum tunneling. Phys. Rep. 1998, 304, 229-354.

69. Truscott, W. Wave functions in the presence of a time-dependent field: Exact solutions and their application to tunneling. Phys. Rev. Lett. 1993, 70, 1900.

70. Büttiker, M.; Landauer, R. Traversal time for tunneling. Phys. Rev. Lett. 1982, 49, 1739.

71. Liu, H. Time-dependent approach to double-barrier quantum well oscillators. Appl. Phys. Lett. 1988, $52,453-455$.

72. Zhu, Z.J. Graphene Geometric Diodes for Optical Rectennas. Ph.D. Thesis, University of Colorado at Boulder, Boulder, CO, USA, 2014. 
73. Han, M.Y.; Özyilmaz, B.; Zhang, Y.; Kim, P. Energy band-gap engineering of graphene nanoribbons. Phys. Rev. Lett. 2007, 98, 206805.

74. Dragoman, D.; Dragoman, M. Geometrically induced rectification in two-dimensional ballistic nanodevices. J. Phys. D Appl. Phys. 2013, 46, 055306.

75. Dirac Equation. Available online: https://en.wikipedia.org/w/index.php?title=Dirac_equation\&oldid= 796044311 (accessed on 21 August 2017).

76. Dragoman, M.; Dinescu, A.; Dragoman, D. On-wafer graphene diodes for high-frequency applications. In Proceedings of the European IEEE Solid-State Device Research Conference (ESSDERC), Bucharest, Romania, 16-20 September 2013; pp. 322-325.

77. Stellingwerff, S.A. Solar Energy Harvesting Using Graphene Rectennas: A Proof-of-Concept Study. Master's Thesis, University of Twente, Enschede, The Netherlands, 2015.

78. Tongay, S.; Lemaitre, M.; Miao, X.; Gila, B.; Appleton, B.; Hebard, A. Rectification at graphene-semiconductor interfaces: Zero-Gap semiconductor-based diodes. Phys. Rev. X 2012, 2, 011002.

79. Dragoman, M.; Aldrigo, M. Graphene rectenna for efficient energy harvesting at terahertz frequencies. Appl. Phys. Lett. 2016, 109, 113105.

80. Torrey, H.; Whitmer, C. Crystal Rectifiers; McGra \v-Hill: New York, NY, USA, 1948; p. 948.

81. Gillespie, D.J.; Ellis, D.P. Modeling nonlinear circuits with linearized dynamical models via kernel regression. In Proceedings of the 2013 IEEE Workshop on Applications of Signal Processing to Audio and Acoustics (WASPAA), New Paltz, NY, USA, 20-23 October 2013; pp. 1-4.

82. Rizzoli, V.; Lipparini, A.; Costanzo, A.; Mastri, F.; Cecchetti, C.; Neri, A.; Masotti, D. State-of-the-art harmonic-balance simulation of forced nonlinear microwave circuits by the piecewise technique. IEEE Trans. Microw. Theory Tech. 1992, 40, 12-28.

83. Briones, E.; Briones, J.; Martinez-Anton, J.; Cuadrado, A.; McMurtry, S.; Hehn, M.; Montaigne, F.; Alda, J.; González, J. Seebeck nanoantennas for infrared detection and energy harvesting applications. In Proceedings of the 2015 9th European Conference on Antennas and Propagation (EuCAP),Lisbon, Portugal, 12-17 April 2015; pp. 1-4.

84. Russer, J.A.; Jirauschek, C.; Szakmany, G.P.; Schmidt, M.; Orlov, A.O.; Bernstein, G.H.; Porod, W.; Lugli, P.; Russer, P. A nanostructured long-wave infrared range thermocouple detector. IEEE Trans. Terahertz Sci. Technol. 2015, 5, 335-343.

85. Periasamy, P.; Guthrey, H.L.; Abdulagatov, A.I.; Ndione, P.F.; Berry, J.J.; Ginley, D.S.; George, S.M.; Parilla, P.A.; O'Hayre, R.P. Metal-insulator-metal diodes: Role of the insulator layer on the rectification performance. Adv. Mater. 2013, 25, 1301-1308.

86. Periasamy, P.; Berry, J.J.; Dameron, A.A.; Bergeson, J.D.; Ginley, D.S.; O’Hayre, R.P.; Parilla, P.A. Fabrication and characterization of MIM diodes based on $\mathrm{Nb} / \mathrm{Nb} 2 \mathrm{O} 5$ via a rapid screening technique. Adv. Mater. 2011, 23, 3080-3085.

87. Mitrovic, I.Z.; Weerakkody, A.D.; Sedghi, N.; Hall, S.; Ralph, J.F.; Wrench, J.S.; Chalker, P.R.; Luo, Z.; Beeby, S. (Invited) Tunnel-Barrier Rectifiers for Optical Nantennas. ECS Trans. 2016, 72, 287-299.

88. Divitt, S.; Novotny, L. Spatial coherence of sunlight and its implications for light management in photovoltaics. Optica 2015, 2, 95-103.

89. Thermophotovoltaic. Available online: https://en.wikipedia.org/w/index.php?title=Thermophotovoltaic\& oldid=793300523 (accessed on 23 August 2017).

90. Lenert, A.; Bierman, D.M.; Nam, Y.; Chan, W.R.; Celanović, I.; Soljačić, M.; Wang, E.N. A nanophotonic solar thermophotovoltaic device. Nat. Nanotechnol. 2014, 9, 126-130.

91. Harder, N.P.; Würfel, P. Theoretical limits of thermophotovoltaic solar energy conversion. Semicond. Sci. Technol. 2003, 18, S151.

92. DeMeo, D.F.; Licht, A.S.; Shemelya, C.M.; Downs, C.M.; Vandervelde, T.E. Thermophotovoltaics: An alternative to and potential partner with rectenna energy harvesters. In Rectenna Solar Cells; Springer: Berlin, Germany, 2013; pp. 371-390.

93. DiMatteo, R.; Greiff, P.; Seltzer, D.; Meulenberg, D.; Brown, E.; Carlen, E.; Kaiser, K.; Finberg, S.; Nguyen, H.; Azarkevich, J.; et al. Micron-gap Thermo Photo Voltaics (MTPV). AIP Conf. Proc. 2004, 738, 42-51.

94. Carozzi, T.; Woan, G. A generalized measurement equation and van Cittert-Zernike theorem for wide-field radio astronomical interferometry. Mon. Not. R. Astron. Soc. 2009, 395, 1558-1568. 
95. Zhu, Z.; Joshi, S.; Grover, S.; Moddel, G. Graphene geometric diodes for terahertz rectennas. J. Phys. D Appl. Phys. 2013, 46, 185101.

96. Bareiß, M.; Krenz, P.M.; Szakmany, G.P.; Tiwari, B.N.; Kalblein, D.; Orlov, A.O.; Bernstein, G.H.; Scarpa, G.; Fabel, B.; Zschieschang, U.; et al. Rectennas revisited. IEEE Trans. Nanotechnol. 2013, 12, 1144-1150.

97. Simmons, J.G. Potential barriers and emission-limited current flow between closely spaced parallel metal electrodes. J. Appl. Phys. 1964, 35, 2472-2481.

98. Goldsmid, H.J. The Seebeck and Peltier effects. In The Physics of Thermoelectric Energy Conversion; 2053-2571, Morgan \& Claypool Publishers: Williston, VT, USA, 2017; pp. 1-1-1-3.

99. Das, V.D.; Soundararajan, N. Size and temperature effects on the Seebeck coefficient of thin bismuth films. Phys. Rev. B 1987, 35, 5990.

100. Li, G.; Han, D.; Yang, F.; Wang, Z.; Pi, Y.; Wang, W.; Xu, S. Linearly enhanced response of thermopower in cascaded array of dual-stripe single-metal thermocouples. Appl. Phys. Lett. 2017, 110, 203505.

101. Huhn, A.K.; Spickermann, G.; Ihring, A.; Schinkel, U.; Meyer, H.G.; Haring Bolívar, P. Uncooled antenna-coupled terahertz detectors with $22 \mu$ s response time based on $\mathrm{BiSb} / \mathrm{Sb}$ thermocouples. Appl. Phys. Lett. 2013, 102, 121102.

102. Szakmany, G.P.; Orlov, A.O.; Bernstein, G.H.; Porod, W.; Bareiss, M.; Lugli, P.; Russer, J.A.; Jirauschek, C.; Russer, P.; Ivrlac, M.T.; et al. Nano-antenna arrays for the infrared regime. In Proceedings of the 18th International ITG Workshop on Smart Antennas (WSA), VDE, Erlangen, Germany, 12-13 March 2014; pp. 1-8.

103. Cuadrado, A.; Alda, J.; González, F.J. Distributed bolometric effect in optical antennas and resonant structures. J. Nanophotonics 2012, 6, doi:10.1117/1.JNP.6.063512.

104. Yang, W.; Zhou, W.; Ma, Z.; Berggren, J.; Hammar, M. Flexible solar cells based on stacked crystalline semiconductor nanomembranes on plastic substrates. In Proceedings of the 2010 Conference on Lasers and Electro-Optics (CLEO) and Quantum Electronics and Laser Science Conference (QELS), San Jose, CA, USA, 16-21 May 2010; p. CML2.

105. Bjorkholm, J.E. EUV lithography-The successor to optical lithography. Intel Technol. J. 1998, 3, 98.

106. Robinson, T.; Yi, D.; Brinkley, D.; Roessler, K.; White, R.; Bozak, R.; Archuletta, M.; Lee, D. Clean and repair of EUV photomasks. Proc. SPIE 2011, 8166, doi:10.1117/12.898503.

107. Slafer, W.D. Techniques for Roll-to-Roll Manufacturing of Flexible Rectenna Solar Cells. In Rectenna Solar Cells; Springer: Berlin, Germany, 2013; pp. 337-369.

108. Slafer, W.D. Tools and Methods for Producing Nanoantenna Electronic Devices. U.S. Patent 9,589,797, 7 March 2017.

109. Bareiß, M.; Kälblein, D.; Krenz, P.M.; Zschieschang, U.; Klauk, H.; Scarpa, G.; Fabel, B.; Porod, W.; Lugli, P., Large-Area Fabrication of Antennas and Nanodiodes. In Rectenna Solar Cells; Springer: Berlin, Germany, 2013; pp. 297-311.

110. Weiler, B.; Nagel, R.; Albes, T.; Haeberle, T.; Gagliardi, A.; Lugli, P. Electrical and morphological characterization of transfer-printed $\mathrm{Au} / \mathrm{Ti} / \mathrm{TiO} \mathrm{x} / \mathrm{p}+-\mathrm{Si}$ nano-and microstructures with plasma-grown titanium oxide layers. J. Appl. Phys. 2016, 119, 145106.

111. Nagel, R.D.; Haeberle, T.; Schmidt, M.; Lugli, P.; Scarpa, G. Large Area Nano-transfer Printing of Sub-50-nm Metal Nanostructures Using Low-cost Semi-flexible Hybrid Templates. Nanoscale Res. Lett. 2016, 11, 143.

(C) 2017 by the authors. Licensee MDPI, Basel, Switzerland. This article is an open access article distributed under the terms and conditions of the Creative Commons Attribution (CC BY) license (http:/ / creativecommons.org/licenses/by/4.0/). 